\title{
X-ray absorption fine structure spectroscopy in nanomaterials
}

\author{
Zhihu Sun, Qinghua Liu, Tao Yao, Wensheng Yan and Shiqiang Wei*
}

X-ray absorption fine structure (XAFS) spectroscopy has been widely used for decades in a wide range of scientific fields, including physics, chemistry, biology, materials sciences, environmental sciences, etc. This review article is devoted to the applications of XAFS in nanomaterials. The basic principles of XAFS are briefly described from the view point of practical application, including its theory, data analysis and experiments. Using selected examples from recent literatures, the power of XAFS in determination of local atomic/electronic structures is illustrated for various nanomaterials, covering metal and semiconductor nanoparticles, catalysts, core/shell structures, ultrathin nanosheets, and so on. The utilization of time-resolved XAFS technique is also briefly introduced, for in-situ probing the nucleation/growth processes of nanomaterials and identifying reaction intermediates of nanostructured catalysts under operando conditions.

\section{INTRODUCTION}

With the emergence of nanoscience and nanotechnology in the late 1980s [1], they have risen dramatically to be one of the most important research areas in the 21st century. The nanoscience and nanotechnology include the study of objects and systems with at least one dimension in the nanometer scale (typically $1-100 \mathrm{~nm}$ ) [2]. The objects studied in such a size range are larger than atoms and small molecules but smaller than macroscopic bulk structures. The dimensions of these systems are comparable to the characteristic length scales that define the overall properties of materials. As a result, many of the physical and chemical properties of the nanometer-scaled materials (nanomaterials) are changed relatively to their bulks, and numerous unique behaviors of the nanomaterials emerge, like the quantum size-effect, quantum confinement effect, near-field optical effects, electron tunneling, and so on. Further development of new and improved nanomaterials requires the ability to control their structure at smaller and smaller scales and complete understanding of their behaviors at nanoscale. With these improved abilities, there will be great potential to create a rich diversity of materials with novel characteristics, functions and applications.
To synthesize nanomaterials in a controllable manner and understand their unique and interesting properties, thorough characterizations of these materials in either static or transient states are essential. Inspired by these motivations, many new techniques (for instance, the atomic force microscope [3] and the scanning tunneling microscope [4]) have been developed and many traditional techniques (like $\mathrm{X}$-ray absorption fine structure (XAFS) reviewed here) have been extensively applied to this rapidly developing field. Many other spectroscopic techniques have been found wide applications in nanomaterials. For example, optical absorption, photoluminescence and Raman spectroscopy have shown their power in investigating the elemental excitations in nanomaterials, but these are beyond the scope of this article, which aims at reviewing the application of XAFS in the determination of atomic structure and electronic structures in nanomaterials.

The last four decades has witnessed the great development of XAFS spectroscopy, as well as its wide applications in nanomaterials. XAFS refers to the oscillatory fine structure of the X-ray absorption coefficient $\mu(E)$ that changes as a function of the incident X-ray photons with energy $E$ beyond the absorption edge $E_{0}$ of a specific atom. Its most outstanding features are the sensitivity to short-range order and element-specificity, which enable them to selectively probe the environments surrounding a specific element in solids, liquids, and even gases $[5,6]$. The development of XAFS as a powerful tool of structure characterization is associated with the development of an effective scattering theory to express the essential physics, and with the availability of synchrotron radiation (SR) light sources that emit tunable and brilliant X-ray photons. Since 1970's, the number of XAFS experiments performed has grown exponentially and XAFS has been routinely used as a local structure probe nowadays. Owing to the building of SR light sources with high-brilliance, numerous measurement methods have been developed to obtain high quality XAFS data under various conditions, such as transmission XAFS for usual bulk material, fluorescence XAFS for trace element, grazing-incidence XAFS for surface research, mag-

National Synchrotron Radiation Laboratory, University of Science and Technology of China, Hefei 230029, China

*Corresponding author (email: sqwei@ustc.edu.cn) 
netic XAFS for spin study, in-situ and operando XAFS for high-temperature/high-pressure/real-reaction investigations, time-resolved XAFS for kinetics studies and even space- resolved XAFS for microzone studies. Nowadays, XAFS has been used in a wide range of scientific fields, including physics, chemistry, biology, materials sciences, environmental sciences, and so on. The structural information provided by XAFS helped to solve many important scientific problems [7-11].

This review will emphasize on the applications of XAFS to the study of nanomaterials. It is organized as follows. We start with the introduction of the basic principles of XAFS, then briefly describe the most commonly used XAFS experimental modes for XAFS measurements, and a general description of the XAFS data analysis procedure. Then selected examples of applications of XAFS in various nanomaterials are introduced, including metal and semiconductor nanoparticles and catalysts, core/shell structures, and ultrathin nanosheets. The last two subsections are focused on the in-situ probing of the nucleation and growth processes of nanomaterials and studies of nanostructured catalysts under operando conditions. Depending on the materials under investigation, XAFS could provide a rich diversity of information, e.g., size-dependent lattice contractions, surface distortions, occupations and distributions of dopants, defect types and dopant valence, chemical reaction progresses, etc. The study on nanomaterials is a rather wide field that a large number of excellent researches have performed, but only a small part of these works could be covered in this review.

\section{BASIC PRINCIPLES OF XAFS}

In the $\mathrm{X}$-ray spectral region, the impinging photons interact with matter through the dominant photoelectric absorption. The basic physical quantity measured in XAFS is the X-ray absorption coefficient $\mu(E)$, which describes how the X-ray absorption of a sample changes as a function of the incident X-ray photons energy $E$. At specific energies, the X-ray photon has sufficient energy to liberate electrons from the low-energy bound states in the absorbing atoms, and causes a sudden increase in the absorption coefficient $\mu(E)$. These energies are called element-specific X-ray absorption edges. Excitation of electrons from $1 s, 2 s, 2 p_{1 / 2}$, and $2 p_{3 / 2}$ corresponds to the $K, L_{1}, L_{2}$, and $L_{3}$ edges, respectively. Across the absorption edge, the X-ray absorption coefficient $\mu(E)$ exhibits a jump, followed by oscillatory structure at higher energies [5]; XAFS then describes the details of X-ray absorption at energies near and above the absorption edge.

Fig. 1 shows the $\mathrm{Cu} \mathrm{K}$-edge spectrum of copper foil, demonstrating the features of a typical XAFS spectrum. The oscillatory structure observed over a wide energy range above the edge, roughly covering typically $30-50 \mathrm{eV}$ until $1000 \mathrm{eV}$ or more, is generally called extended X-ray absorption fine structure (EXAFS) spectroscopy. The spectral region extending the energy range from the absorption edge (or a little below) to $30-50 \mathrm{eV}$ above it, is commonly referred to as X-ray absorption near-edge structure (XANES). The distinction between XANES and EXAFS is somewhat arbitrary and there is no fundamental difference in the physics giving rise to the fine structures. However, their complexity and analysis methods are quite different. In the EXAFS region, the excited photoelectrons have high kinetic energy and are weakly affected by the neighboring atoms' potential; therefore, the single-scattering processes of the photoelectrons by the neighbors are dominant in most cases. In contrast, in the XANES region, the photoelectron energy is close to the absorption edge and the neighboring atoms' potential has significant influence on the photoelectron scattering; consequently, the multiple-scattering processes give a great contribution to the fine structure.

In the X-ray absorption process, when an incident X-ray photon with energy $E$ higher than the absorption edge $E_{0}$ of a specific atom A (as shown in Fig. 2) is absorbed, the $\mathrm{X}$-ray photon has the sufficient energy to emit an outgoing core-level electron with a wavenumber $k=\left[2 m\left(E-E_{0}\right) / \hbar^{2}\right]^{1 / 2}$ ( $\hbar$ is the Planck's constant and $m$ is the mass of an electron). If there is a neighboring atom $\mathrm{B}$ as schematically described in Fig. 2, its potential barrier of the electron shells scatters the excited photoelectron wave back to the absorbing atom A (incoming waves). The incoming photoelectron waves interfere with the outgoing waves and thus the interference changes the X-ray absorption coefficient of atom A, resulting in an oscillatory structure in the X-ray absorption coefficient: this is the origin of XAFS.

In the last 40 years, XAFS has evolved into a power-

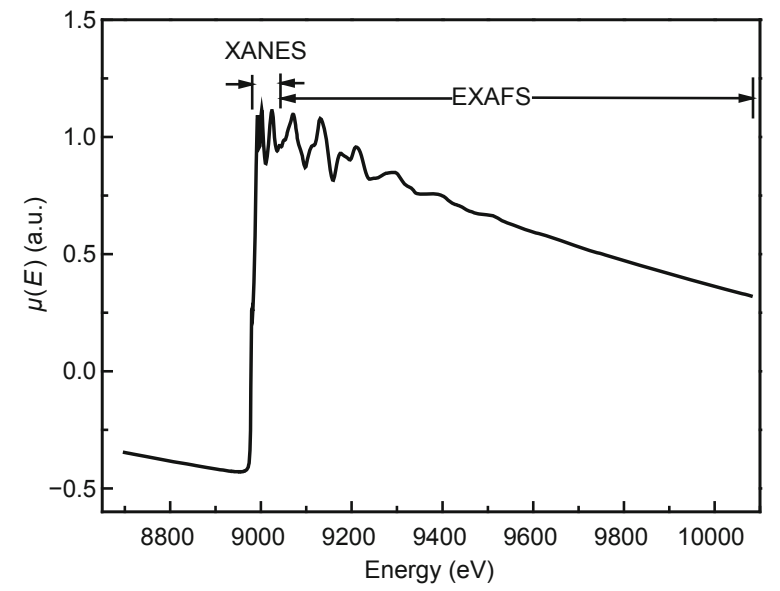

Figure $1 \mathrm{Cu} K$-edge XAFS spectrum for copper foil highlighting the EXAFS and XANES spectral region. 


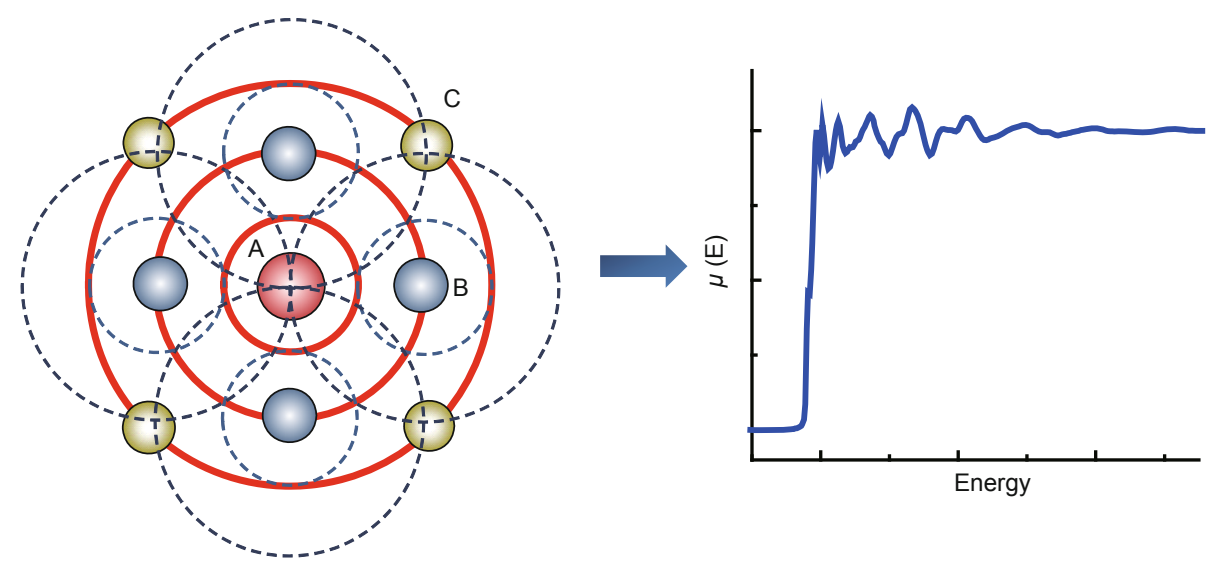

Figure 2 Pictorial view of the origin of XAFS: scattering of an outgoing photoelectron wave by neighboring atoms of the absorbing atom A. The solid circles stand for the outgoing photoelectron waves, and the dashed lines stand for the scattered waves.

ful structural characterization tool. It possesses striking advantages such as element-specificity, sensitivity to the short-range order (typically several $\AA$ ) and chemical state [5]. It can provide quantitative structural information at an atomic scale about the local environment around the absorbing atom, i.e., near-neighbor species and distance, symmetry, coordination number and fluctuation in bond distance. More specifically, EXAFS is sensitive to the distance, coordination number and species of the atoms immediately surrounding the absorbing atoms. XANES can distinguish the electronic state and the three-dimensional coordination geometry (including symmetry, atomic types, bond angles and distances). The sensitivity to the shortrange order and element-specificity endow XAFS a unique capability to determine the local structure for a diversity of systems, no matter the studied sample is in solid (crystalline or amorphous), liquid or even gas state.

Despite the power of XAFS as a local structure probing technique, it also has limitations. First, it can only afford the ensemble-averaged structure information around the absorbing atom, but is difficult to discriminate the target elements mixed in different phases or located at different special positions. Second, its sensitivity to the local structure is limited to a radial range within the first a few coordination shells.

XAFS has a long history of more than 90 years since it was first observed by Friche and Hertz in 1920 [12,13]. However, this phenomenon has long been plagued by controversial physical interpretations. In 1931, Kronig [14] originally interpreted the XAFS oscillations in crystals as a density-of-states effect in terms of the so-called longrange order (LRO) theory. But attempts to match EXAFS peaks with predictions of LRO theories proved unsatisfactory, and afterwards in 1932, Kronig $[15,16]$ presented an alternative theory for small molecules, in which the oscillatory structure in EXAFS was attributed to the influence of neighboring atoms on the transition matrix element in the golden rule, i.e., a short range-order (SRO) effect. The debate between the LRO and SRO theories remained for 40 years until in 1971, Sayers, Stern and Lytle performed the $k$-space Fourier transform to separate peaks corresponding to various atomic shells [17]. This is a milestone in the development story of XAFS. Since then it has been widely recognized that the oscillations in the X-ray absorption spectra, more than just a physical phenomenon, could be used as a quantitative structure-determination technique.

In the framework of the single-scattering which assumes that the outgoing wave is scattered back only once by the neighboring atoms (e.g., the single-scattering path $A \rightarrow B \rightarrow A$ in Fig. 2), the EXAFS oscillation function $\chi(k)$ for randomly oriented polycrystalline or solution samples is expressed by:

$$
\begin{aligned}
\chi(k)= & \sum_{j} \frac{N_{j} S_{0}^{2} f_{j}(k)}{k} \int_{0}^{\infty} \frac{g(R)}{R^{2}} \mathrm{e}^{-2 R / \lambda(k)} \\
& \times \sin \left[\left(2 k R+\delta(k)+2 \varphi_{C}(k)\right] \mathrm{d} R .\right.
\end{aligned}
$$

The contributions from atoms of the same atomic number and at similar distances from the absorbing atom may not be resolvable from each other, and the atoms must be conceptually grouped together into "coordination shells" and $N_{j}$ is the number of atoms in the $j$-th coordination shell. In Equation (1), $S_{0}{ }^{2}$ is the amplitude reduction factor, $R$ is the distance between the absorbing and scattering atoms, $g(R)$ is the pair distribution function of the scattering atom, $\lambda(k)$ is the mean free path of the excited photoelectron, $f_{\mathrm{j}}(k)$ and $\delta(k)$ are the backscattering amplitude and scattering phase shift of the scattering atom, respectively, $\varphi_{\mathrm{C}}(k)$ is phase-shift of the absorbing atom. The amplitude reduction factor $S_{0}{ }^{2}$ is weakly dependent on the absorber elements and is typically in the range of $0.7-1.0$ [18]. The $\mathrm{e}^{-2 R / \lambda}$ term is due to the limited lifetime of the core hole $(\sim 1$ fs) and the limited range (within $10 \AA$ ) of the excited pho- 
toelectron within the matrix, both of which have the effect of damping the oscillation amplitude and are the origin of the local sensitivity of EXAFS.

Equation (1) is applicable to all systems with disorder degrees ranging from low to high. Especially for the low disorder systems, the $g(R)$ function can be represented by a Gaussian distribution,

$$
g(R)=\left(2 \pi \sigma^{2}\right)^{-\frac{1}{2}} \exp \left[-\frac{\left(R-R_{j}\right)^{2}}{2 \sigma^{2}}\right] .
$$

Substituting Equation (2) into Equation (1) gives

$$
\begin{aligned}
\chi(k)= & \sum_{j} \frac{N_{j} S_{0}^{2} f_{j}(k)}{k R_{j}^{2}} \mathrm{e}^{-2 k^{2} \sigma_{j}^{2}} \mathrm{e}^{-2 R_{j} / \lambda_{j}(k)} \\
& \times \sin \left[2 k R_{j}+\delta_{j}(k)+2 \varphi_{C}(k)\right],
\end{aligned}
$$

which is commonly called the standard EXAFS formula. The $\sigma^{2}$ term, known as the Debye-Waller factor, is the mean square variation in the interatomic distance $R$. The $\mathrm{e}^{-2 k^{2} \sigma_{j}^{2}}$ term accounts for the effects of dynamic (thermal) vibration and configuration (structural) disorders which smear out the EXAFS oscillations at high $k$ region.

For systems with moderately higher disorder, like hightemperature samples and surfaces, the Gaussian distribution fails to represent the pair distribution function, and the asymmetry in the pair distribution must be considered. For instance, thermal expansion cannot be described within a harmonic oscillator but requires anharmonic interatomic potential. In such a case, a widely used model-independent method is cumulant expansion that takes the inharmonic pair into account $[19,20]$. In the systems with even higher disorder, such as liquid or amorphous materials, some other model-dependent approximation has been considered; for example, a weighted exponential distribution function could be used to account for the structural disorder [21,22].

The EXAFS Equation (1) is derived based on the single-scattering approximation, which assumes that the outgoing photoelectron wave is only scattered once by the neighboring atoms before returning to the absorbing atoms. But in reality the photoelectron is scattered by more than one neighboring atom and these multiple-scattering (MS) events also contribute to the interference phenomenon. If the multiple-scattering contributions are small, the single-scattering approximation would be sufficient to describe the EXAFS process. For instance, in collinear configuration where three or more atoms are aligned or almost aligned, the multiple-scattering EXAFS contributions can even be larger than the high shell single-scattering contribution because of the shadowing or focusing effect. Therefore, in order to avoid poor or misleading results in the extracting the structural information beyond the first shell, attention to the multiple-scattering events involving different shell atoms should be paid.

Historically, the theoretical treatment of the multiplescattering EXAFS in terms of effective path length was first proposed in 1975 by Lee and Pendry who took advantage of Fourier transform in the EXAFS data analysis [23]. Its main advantages are its computational efficiency and accuracy; moreover, it provides a geometrical parametrization and interpretation of EXAFS, which is ideal for analyzing interatomic distances and other structural quantities. Later, the path-by-path XAFS theory was greatly developed by Rehr and Albers who proposed a separable representation of the Green's-function propagators [24] to overcome all the computational difficulties of the multiple-scattering expansion. For an $N$-leg path $\Gamma$ with scatterers at $\boldsymbol{R}_{1}, \boldsymbol{R}_{2}, \ldots$, $\boldsymbol{R}_{N}=\boldsymbol{R}_{0}$, the result for the EXAFS amplitude $\chi_{\Gamma}$ can be recast exactly in the form of the standard EXAFS equation, but with an effective scattering amplitude $f_{\text {eff }}$,

$$
\chi_{\Gamma}(k)=\operatorname{Im}\left[\frac{f_{\text {eff }}(k)}{k R_{\text {eff }}^{2}} \mathrm{e}^{i\left(2 k R_{\text {eff }}+\delta_{1}(k)\right)} \mathrm{e}^{-2 k^{2} \sigma^{2}}\right] .
$$

The implementation of these algorithms into the $a b$ initio EXAFS code known as FEFF-named after the effective scattering amplitude $f_{\text {eff }}$ in the theory-has made accurate calculations of high-order multiple-scattering EXAFS in general materials routine [25]. By using FEFF code, the effective amplitude function $f_{\text {eff }}$, phase shift function $\delta_{1}(k)$ and the mean free path of electrons $\lambda(k)$ can be calculated for both single- and multiple-scattering paths. For more details on the multiple-scattering FEFF theory, the readers are referred to the excellent review article written by Rehr and Albers [26]. So far, various advancements in the theoretical models have been obtained such as full multiple-scattering (FMS) [27], self-consistent field and fully relativistic calculations [28] especially for a quantitative analysis of the near-edge spectroscopy. The EXAFS theory is implemented in various $a b$ initio codes like CONTINUUM [29], EXCURV [30], FEFF [25], GNXAS [31], WIEN2k [32], NSRL-XAFS [33], and others [34-36].

\section{XAFS EXPERIMENTAL TECHNIQUES AND DATA ANALYSIS}

\section{XAFS experimental techniques}

The objective of a XAFS measurement is to record an energy-dependent quantity that is directly proportional to the absorption coefficient $\mu(E)$ of the atom of interest. Since the magnitude of the XAFS oscillatory function is typically on the order of $10^{-2}$ of the edge-jump and decreases with increasing energy, a fairly precise and accurate measurement scheme is required to collect high quality data. The main experimental key points are the availability of a tun- 
able X-ray source with high photon flux and high energy resolution, and high performance detectors for recording the XAFS data.

The modern XAFS measurements are overwhelmingly performed on synchrotron radiation light sources, which deliver the highly brilliant X-rays with a wide range of wavelengths. In order to select monochromatized photons from the incident beam with a continuous wavelength, a double-crystal monochromator is commonly used (Fig. 3), which selects the wavelength of the incident beam $\lambda$ to the Bragg angle $\theta$ according to the Bragg's equation $2 d \sin \theta=$ $n \lambda$, with $d$ being the interplanar spacing of the crystal. The most commonly used crystal planes are $\mathrm{Si}(111)$ and $\mathrm{Si}(311)$ with $d$ values of 3.1356 and $1.6375 \AA$, respectively. The higher-harmonic contamination also fulfills the Bragg's equation, but they will cause serious systematic error to the spectrum and should be minimized. This can easily be done by either using a mirror or detuning the two crystals of the monochromator.

Depending on different schemes of detecting $\mu(E)$, various XAFS experimental modes have been developed. Among them the transmission and fluorescence modes as shown schematically in Fig. 3 are the most frequently used and will be briefly described. More details on the measurement requirements for getting good XAFS data could be found in many available review papers or book chapters $[5,6,37]$.

\section{Transmission mode}

The transmission mode is the most convenient for collecting XAFS data with good data quality. It relies on the measurement of monochromatic photons intensities incident on $\left(I_{0}\right)$ and transmitted by $\left(I_{t}\right)$ a sample of thickness $d$. The incident and transmitted beam intensities $I_{0}$ and $I_{t}$ are related by $I_{\mathrm{t}}=I_{0} \mathrm{e}^{-\mu(E) d}$, then the absorption coefficient is calculated as $\mu(E) d=-\ln \left(I_{\mathrm{t}} / I_{0}\right)$. The intensities of $I_{0}$ and $I_{\mathrm{t}}$ are usually monitored by ion chambers. Care must be taken to make sure that the two ion chambers are working in the linear region, and the choice of their lengths and gas compositions is essential.

Transmission mode XAFS is mostly suitable for concen- trated samples in which the element of interest is the major component $(>10 \%)$. A crucial requirement for the sample is that it must be uniform and pinhole-free. Typically, the sample thickness $d$ should be optimized to assure the edge jump $\Delta \mu d \approx 1$, where $\Delta \mu$ is the difference of the absorption coefficient before and after the absorption edge. If this is satisfied, the "thickness effects" [38] could be avoided and the data could have a good signal/noise ratio. This requires that the grain sizes for powder samples should be smaller than or comparable to the absorption length of the material.

For a single substance, the absorption coefficient $\mu$ $\left(\mathrm{cm}^{-1}\right)$ is related to the absorption cross $\sigma\left(\mathrm{cm}^{2} \mathrm{~g}^{-1}\right)$ by $\mu=$ $\rho \sigma$, where $\rho\left(\mathrm{g} \mathrm{cm}^{-3}\right)$ is the mass density. For a compound consisting of different elements, the total absorption coefficient $\mu$ is calculated as:

$$
\mu \approx \sum_{i} \rho_{i} \sigma_{i}=\rho_{M} \sum_{i} \frac{m_{i}}{M} \sigma_{i}=\rho_{N} \sum_{i} \frac{n_{i}}{N} \sigma_{i},
$$

where $\rho_{M}$ is the mass density of the material as a whole, $\rho_{N}$ is the number density of the material as a whole, and $m_{i} / M$ and $n_{i} / N$ are the mass fraction and number fraction of element $i$, respectively. Energy-dependent cross sections $\sigma(E)$ of all elements have been tabulated and could be found in literatures $[39,40]$.

Here are some examples of the optimal thickness for different materials. For Fe metal, $\rho=7.86 \mathrm{~g} \mathrm{~cm}^{-3}$, the absorption cross sections exactly before and after the $K$ absorption edge $(7112 \mathrm{eV})$ are 407.6 and $53.3 \mathrm{~cm}^{2} \mathrm{~g}^{-1}$; therefore across the absorption edge $\Delta \mu=\rho \Delta \sigma=7.86 \times(407.6-53.3)$ $=2784.8 \mathrm{~cm}^{-1}$. The optimal thickness $d$ satisfying $\Delta \mu d \approx 1$ is then $d=1 / 2784.8=3.6 \mu \mathrm{m}$. For iron oxide, such as $\mathrm{Fe}_{3} \mathrm{O}_{4}$, calculation using Equation (5) gives the optimal thickness of $7.5 \mu \mathrm{m}$. For heavier element like Ag metal, the optimal thickness is much larger, $21 \mu \mathrm{m}$ at $\mathrm{Ag} \mathrm{K}$-edge. For dilute solutions, $d$ is typically in the millimeter range. But in the soft X-ray region, the optimal thickness is much smaller. For example, at $\mathrm{Al} \mathrm{K}$-edge the optimal thickness for $\mathrm{Al}$ metal is only $1.0 \mu \mathrm{m}$; such a too small thickness makes it practically very hard to prepare a uniform sample and the transmission technique usually cannot be applied in the

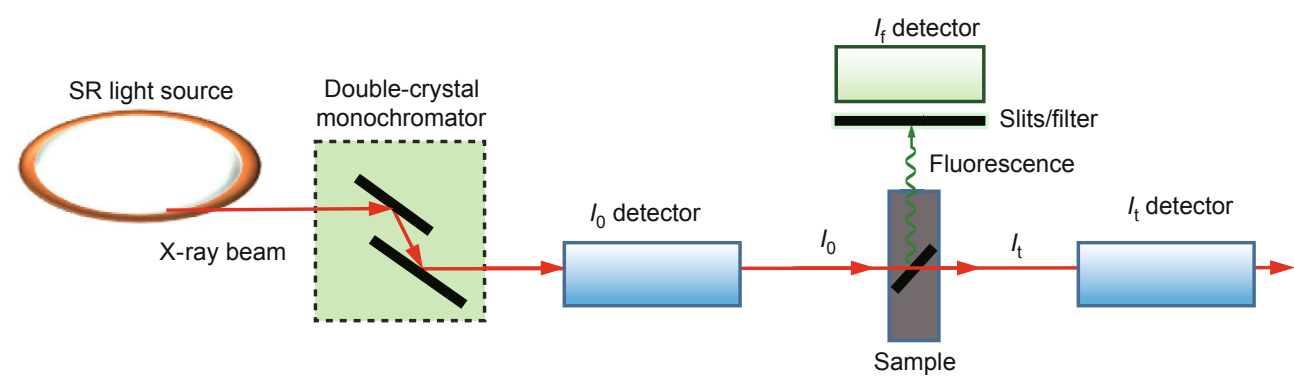

Figure 3 The schematic XAFS experimental setup in a transmission or fluorescence mode. 
soft X-ray region.

\section{Fluorescence yield mode}

The requirement of $\Delta \mu d \approx 1$ in a transmission experiment could be hardly fulfilled for a dilute sample, like the lightly doped samples such as diluted magnetic semiconductors and thin films grown on substrates. Otherwise, the background absorption due to the matrix or substrate would be very large and the transmitted beam intensity would be exponentially attenuated, resulting in very bad quality of the data. To reduce the background absorption, selectively recording the atomic-specific signals is a good choice. Among them, monitoring the $\mathrm{X}$-ray fluorescence is the most preferred, since under proper conditions the fluorescence yield is proportional to the absorption efficient (see Equations (7) and (8) below).

In a fluorescence XAFS measurement, the X-rays emitted from the sample will include the fluorescence line of interest, fluorescence lines from other elements in the sample, and both elastic and inelastic (Compton) scattering $\mathrm{X}$-rays. To make good fluorescence XAFS measurements, it is desirable to collect the available fluorescence signal as much as possible. Although in general the fluorescence is emitted isotropically, the scatter is not emitted isotropically because the X-rays from a synchrotron are polarized in the plane of the synchrotron. This means that elastic scatter is greatly suppressed at $90^{\circ}$ to the incident beam in the horizontal plane. Therefore, fluorescence detectors are normally placed in the horizontal plane at a right angle to the incident beam, and the sample is placed at $45^{\circ}$ to both the incident beam and the fluorescence detector, as shown schematically in Fig. 3. In this geometry, the fluorescence intensity $I_{\mathrm{f}}(E)$ accepted by a detector with a solid angle $\Omega / 4 \pi$ is:

$$
\begin{aligned}
I_{\mathrm{f}}(E)= & \frac{I_{0} \gamma \Omega}{4 \pi} \frac{\mu_{\mathrm{A}}(E)}{\mu_{\text {tot }}(E)+\mu_{\mathrm{b}}\left(E_{\mathrm{f}}\right)} \\
& \times\left\{1-\exp \left[-\left(\mu_{\mathrm{tot}}(E)+\mu_{\mathrm{b}}\left(E_{\mathrm{f}}\right)\right) d\right]\right\} .
\end{aligned}
$$

Here $\gamma$ is the fluorescence efficiency, $d$ is the sample thickness, $E_{\mathrm{f}}$ is the energy of the fluorescent photons, $\mu_{\mathrm{A}}(E)$ is the absorption from the element of interest, $\mu_{\mathrm{b}}(E)$ is the background absorption coefficient of all the other elements, and $\mu_{\text {tot }}(E)=\mu_{\mathrm{A}}(E)+\mu_{\mathrm{b}}(E)$ is the total absorption coefficient. Because $\mu_{\text {tot }}(E)$ depends on $\mu_{\mathrm{A}}(E)$, in general $I_{\mathrm{f}}$ is not proportional to $\mu_{\mathrm{A}}(E)$. But in two limiting cases this proportion holds:

Thin samples (typically below $300 \mathrm{~nm}$ ), in which the series expansion of the exponential in Equation (6) can be truncated at the term linear in $d$, i.e., $-\left(\mu_{\text {tot }}(E)+\mu_{\mathrm{b}}\left(E_{f}\right)\right) d$ $<<1,1-\exp \left[-\left(\mu_{\text {tot }}(E)+\mu_{\mathrm{b}}\left(E_{\mathrm{f}}\right)\right) d\right] \approx\left(\mu_{\mathrm{tot}}(E)+\mu_{\mathrm{b}}\left(E_{\mathrm{f}}\right)\right) d$, and then

$$
I_{\mathrm{f}}(E)=I_{0} \gamma(\Omega / 4 \pi) d \mu_{\mathrm{A}}(E) \propto I_{0} \mu_{\mathrm{A}}(E) .
$$

Thick samples with a dilute concentration of the element of interest, in which $\mu_{\text {tot }}(E)>>\mu_{\mathrm{A}}(E)$ and 1-exp [$\left.\left(\mu_{\text {tot }}(E)+\mu_{\mathrm{b}}\left(E_{f}\right)\right) d\right] \approx 1$. Then Equation (6) reduces to

$$
I_{\mathrm{f}}(E)=I_{0} \gamma(\Omega / 4 \pi) \frac{\mu_{\mathrm{A}}(E)}{\mu_{\mathrm{tot}}(E)} \propto I_{0} \mu_{\mathrm{A}}(E) .
$$

If neither of the above two conditions was met, a strong suppression of features in the spectrum could be observed. Hence, use of a thick, concentrated sample results in severely distorted XAFS spectra. Such a phenomenon, known as the "self-absorption" effect of the fluorescence data, should be avoided or corrected [41,42].

In the fluorescence mode, to collect only the fluorescence line of interest, and completely suppress the scattering signals and other fluorescence lines are desirable. This would greatly decrease the background intensity, and increase the signal/noise ratio. The intensity of the scattering can be reduced by an order of magnitude by inserting an $\mathrm{X}$-ray filter that has the absorption edge between the fluorescence line of interest and the elastic scattering peak. A thin metal foil or a homogeneous powder containing the $Z-1$ element ( $Z$ is the atomic number of the element of interest) is conventionally used as a filter. For example, to measure Co $K$-edge fluorescence XAFS data, a 3- or $6-\mu \mathrm{m}$ thick $\mathrm{Fe}_{2} \mathrm{O}_{3}$ filter is usually used. Besides, to avoid re-radiation from the filter itself, a set of slits (Soller slits) is often used to preferentially collect the emission from the sample and block the emission from the filter.

The fluorescence X-ray detector could be a Lytle detector [43], which is also an ionization chamber, but it cannot discriminate the fluorescence of interest from the background. To suppress the background intensity, detectors with energy-discriminating ability, such as high-purity Ge or Si solid-state detector (SSD) and silicon drift diode (SDD) detector, have been developed and used. The energy resolution of these detector is typically in the range of $150-200 \mathrm{eV}$ at $6 \mathrm{keV}$, as a result of a balance between the counting rate and the background removal. The maximum counting rate of these detectors is limited by the detector electronics, which can be enhanced by an order of magnitude by assembling multiple detector elements with a close packing geometry. The dilute limit of the fluorescence XAFS measurements is determined by the detector counting rate and the incident beam intensity. A state-of-the-art Ge pixel array detector (PAD) with 100 segments has been developed to gain high throughput measurement of fluorescence yield by Oyanagi et al. [44]. Because both the incident and the detected signals in the fluorescence mode are penetrating X-rays, fluorescence XAFS is the most suitable choice to study bulk samples. It could also be a surface-sen- 
sitive method by using a grazing-incidence geometry [45].

\section{EXAFS data analysis}

The aim of EXAFS data processing and analysis is to extract structural parameters such as coordination numbers, interatomic distances, disorder degrees and sometimes, types of atoms, in various coordination shells for an "unknown" sample. The accuracy of EXAFS in obtaining these structural information depends heavily on the standard phase-shift and the amplitude functions which are derived from either experimental reference compounds or theoretical calculations. In general, the EXAFS data analysis procedure consists of the following steps: deglitching, preedge background removal, normalization, conversion to
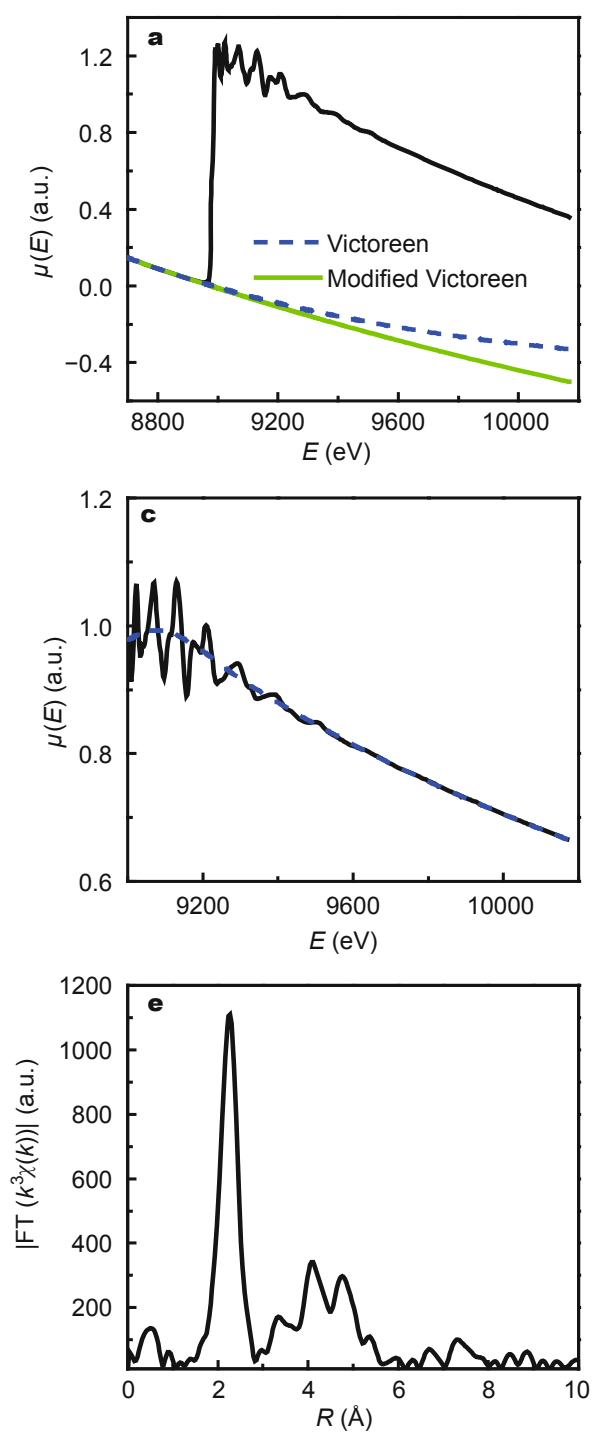

$k$-space, Fourier transform to $R$-space and reverse Fourier transform (Fig. 4). The order of these steps is not critical and not all the steps are necessary. In the following we will take the EXAFS spectrum of $\mathrm{Cu}$ foil as an example to show this general procedure in detail, addressing some points that need special attention.

Deglitching The raw EXAFS spectra sometimes contain abnormally sharp data points called "glitches". Their origin might be diffractions from the crystal monochromator or from the single crystal samples (including crystal substrates), or multi-electron excitations. These glitches might be many times larger than the real EXAFS signal and can cause "spectral leakage" in the Fourier transform curves, and must be removed before further processing. The com-
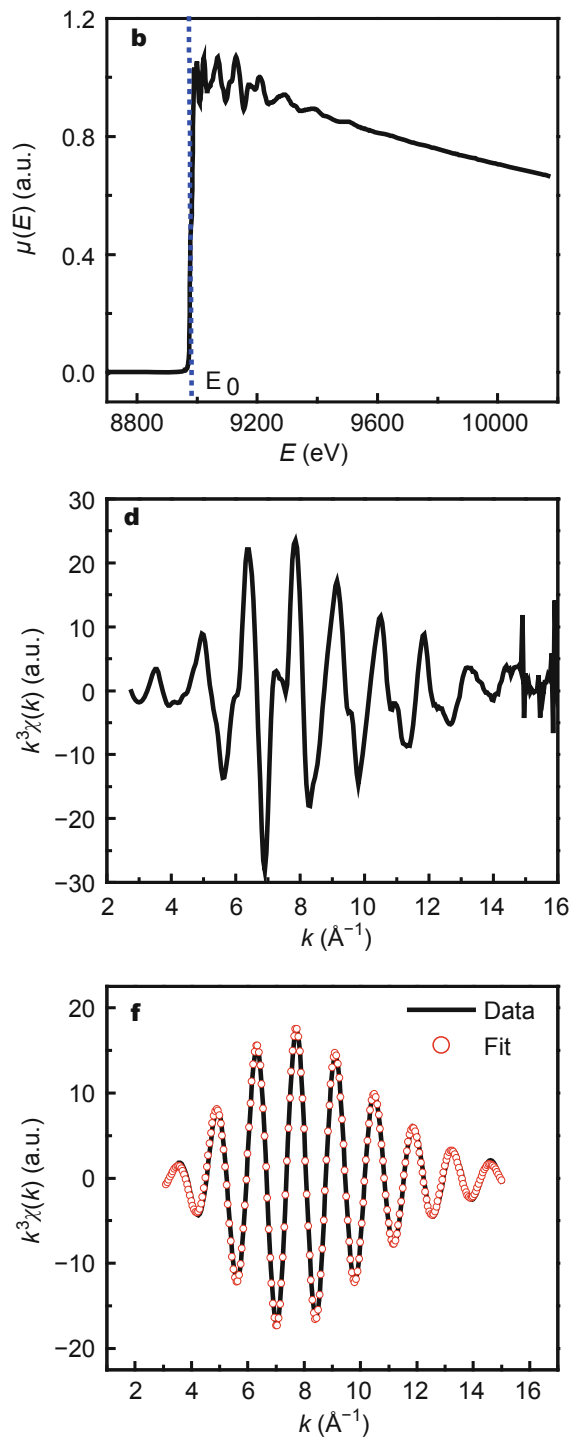

Figure 4 Typical EXAFS data processing and analysis procedure: (a) pre-edge background removal, (b) normalization, (c) curve fitting of $\mu_{0}$, (d) conversion to $k$-space and weighting, (e) Fourier transform to $R$-space, and (f) reverse Fourier transform and fitting in the $k$-space. 
mon deglitching technique is smooth interpolation of the data on both sides of the glitch.

Pre-edge background removal The most interesting region in the EXAFS data is the part above the absorption edge, and the pre-edge background absorption should be removed. This generally involves a fitting of the data before the edge to some functional form and extrapolating this function into the post-edge data. A traditional function used for the fitting is the Victoreen empirical formula $\mu_{\mathrm{v}}(E)$ $=a E^{-3}+b E^{-4}$ or the modified Victoreen formula $\mu_{\mathrm{v}}(E)=$ $a E^{-3}+b E^{-4}+c$, as exemplified in Fig. 4 a.

Data normalization This step is taken to normalize the absorption coefficient to that of one single atom, for annihilating the difference in the samples. The most widely used normalization method is to use the relation,

$$
\chi(E)=\frac{\mu(E)-\mu_{0}(E)}{\Delta \mu\left(E_{0}\right)} .
$$

Here $\mu_{0}(E)$ is the atomic part of the absorption, $\Delta \mu\left(E_{0}\right)$ is the height of the edge-step, and $E_{0}$ is the photoelectron excitation threshold energy. This $E_{0}$ is close to, but somewhat different from the absorption edge of the element under investigation, as it is affected by the chemical state or valence. There is no general way to determine this threshold energy, and in practice it is chosen as an inflection point (where the $\mathrm{d} \mu(E) / \mathrm{d} E$ reaches maximum) or the middle point of the edge jump. The errors in the determination of $E_{0}$ could further be compensated in the curve-fitting procedure, where a parameter $\Delta E$ will be optimized.

A common method of determining $\Delta \mu\left(E_{0}\right)$ is to fit the data within a few hundreds eV below and above the absorption edge with low order polynomials, then extrapolate them to the $E_{0}$ point (Fig. $4 \mathrm{~b}$ ). The difference between these two curves at $E_{0}$ is the step $\Delta \mu\left(E_{0}\right)$, and the resulting EXAFS spectrum is then normalized to an edge jump of 1 .

Curve fitting of $\mu_{0}(E)$ Till now, the normalized $\mu(E)$ data still contain the atomic absorption $\mu_{0}(E)$ that must be subtracted out. In general, $\mu_{0}(E)$ is neither measurable nor can be calculated theoretically. Practically, a least-squares cubic spline functions fitting is used to obtain $\mu_{0}(E)$ (Fig. 4c). In doing this the data are divided into several regions (the divided points are called nodes), and separate cubic polynomials are fit to the data over each region. The cubics are constrained so that the curves in each region are connected continuously (the values, the first and second derivatives match at the junctures). The atomic background absorption is then removed to reveal the EXAFS oscillatory curve $\chi(E)$.

Conversion to $k$-space and weighting For performing the subsequent Fourier transform (FT), the E-space EXAFS data $\chi(E)$ must be converted to $\chi(k)$ in the $k$-space, according to $k=\left[2 m\left(E-E_{0}\right) / \hbar^{2}\right]^{1 / 2}$. At the same time, the $\chi(k)$ data are generally weighted with some power of $k^{n}(n=1,2,3)$ to compensate for the decay of the waves with extended $k$ (Fig. $4 \mathrm{~d}$ ) as well as to diminish the ambiguity of $E_{0}$. The choice of the $k$-weighing power is arbitrary, but there are still some general comments. The $k^{3}$-weight is most frequently used for analysis of EXAFS data contributed by heavy atoms coordination, and the $k$ - or $k^{2}$-weight is more suitable for lighter coordination atoms in order to enhance their contributions maximized in the low $k$-region.

Fourier transform to $R$-space The next step is to perform Fourier transform (FT) of the $k^{n} \chi(k)$ function to the $R$-space (Fig. 4e). This is a very important step, since the EXAFS oscillations are the sum of different sinusoidal functions of many shells as in Equation (3) and the FT technique could separate their contributions. It must be addressed that in the FT curve, the peak position is not identical to the interatomic distance as the real Radial Distribution Function (RDF) does, but shifts typically by $0.3-0.5 \AA$ for the first shell atoms to the lower $R$-side, due to the phase-shift in the EXAFS equation.

Reverse Fourier transform The following step is to reversely transform the $R$-space peak corresponding to a single shell to $k$-space (Fig. 4f). Then the oscillation of this specific shell is filtered from the overall spectrum, which allows extracting the structural information of that shell through a curve-fitting procedure. This step is not necessary, since nowadays the curve-fittings are more commonly done in the $R$-space.

Fitting procedure Once the single-shell oscillation has been isolated, the quantitative structural parameters can be obtained through a curve-fitting procedure according to the EXAFS standard formula Equation (3). Besides the structural parameters $N, R, \sigma^{2}$ that appear explicitly in the EXAFS equation, there is another parameter $\Delta E_{0}$ to be optimized. This parameter at least plays dual roles of correcting the errors in determining the threshold of the absorption energy and compensating the inaccuracy of phase-shift. Before curve-fitting the amplitude and the phase-shift functions should be known. Thanks to the development of the accurate $a b$ initio calculations of FEFF code, nowadays the theoretical amplitude and the phase-shift are more routinely used than the experimental ones.

There are various algorithms for fitting the filtered experimental EXAFS function, among which the leastsquares algorithm is most commonly used. However, the least-squares algorithm suffers from some drawbacks, for example, many local minima in the sum of squares function may exist, and the obtained structural parameters are often physically impossible. To overcome this problem, some new algorithms have been developed, like the simulated annealing method implemented in the USTCXAFS3.0 software package developed by our group [33]. Shown in 
Table 1 are the structural parameters of $\mathrm{Cu}$ foil extracted from EXAFS fitting with USTCXAFS3.0 as an example.

For an EXAFS spectrum with a good signal/noise ratio, typically the accuracy of the first-shell structural parameter determination can be: interatomic distance $R, \pm 0.01 \AA$ or better; coordination number $N$, within $\pm 10 \%$; and Debye-Waller factor $\sigma^{2}$ in the Gaussian approximation, within $\pm 0.0005 \AA^{2}$. For higher-shells, the accuracy of the obtained structural parameters is usually lower. In practice, one problem in the multi-shell fitting is the correlation among various parameters. For instance, both $N$ and $\sigma^{2}$ are related to the oscillation amplitude and hence are strongly correlated; if both are treated as free parameters, their accuracy would be decreased. It often occurs that when too many parameters are to be fitted, the correlations may force the fitting results to converge on values without physical meaning. Therefore, reasonable constraints between parameters are necessary.

\section{XAFS APPLICATIONS IN NANOMATERIALS}

The XAFS technique, due to its element-specific feature and powerful probing ability for atomic/electronic structures in condensed matter, has been applied extensively in nanomaterials fields. The following subsections will be focused on the applications of XAFS to the study of various nano-structured materials. They cover metal and semiconductor nanoparticles and catalysts, core/shell structures, ultrathin nanosheets, as well as the in-situ probing of the nucleation and the growth processes of nanomaterials and the studies of nanostructured catalysts under operando

Table 1 The structure parameters of $\mathrm{Cu}$ foil from a curve fitting

\begin{tabular}{ccc}
\hline Parameter & Real value & Fitting result \\
\hline$R(\AA)$ & 2.55 & $2.55 \pm 0.01$ \\
$N$ & 12 & $12.0 \pm 0.4$ \\
$\sigma^{2}\left(\AA^{2}\right)$ & - & $0.0086 \pm 0.0003$ \\
$\Delta E_{0}(\mathrm{eV})$ & - & $-4.4 \pm 0.5$ \\
\hline
\end{tabular}

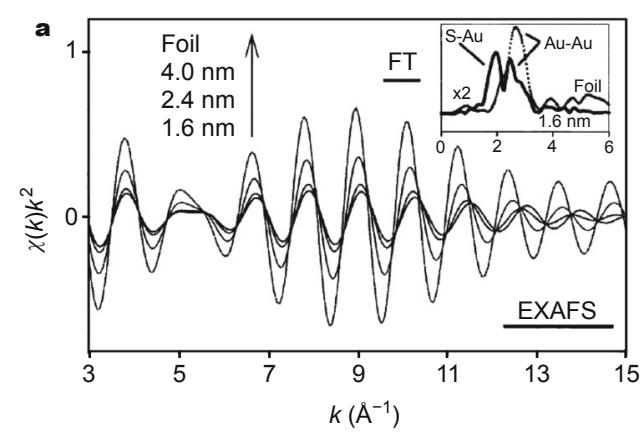

conditions. The content will be arranged according to the materials systems, with focus on the XAFS-revealed atomic and electronic structural features that play important roles in understanding the structure-function relationship.

\section{Metal nanocrystals and nanoclusters}

Monodisperse nanocrystals have fascinating size-dependent optical, electronic, catalytic and chemical properties which make them very promising in the development of new generations of catalysts, sensors, and optoelectronic devices [46-49]. To understand the interplay between the size and the surface effects that determines the structure and the electronic properties of gold nanocrystals, Zhang and Sham [50] conducted a study on a series of alkanethiol-capped Au nanocrystals prepared by the colloidal synthesis techniques in the presence of thiol. The size of the thiol-capped nanocrystals were determined by the ratio of the Au precursor $\left(\mathrm{HAuCl}_{4}\right)$ and the thiol; Au:S ratios of 1:3, $1: 1$, and $6: 1$ yielded nanocrystals of $1.6,2.4$, and $4.0 \mathrm{~nm}$, respectively. The $\mathrm{Au} \mathrm{L}_{3}$-edge EXAFS data show a size-dependent lattice contraction, as apparent in the reverse Fourier transform curves of the first $\mathrm{Au}-\mathrm{Au}$ pairs where the oscillation becomes progressively farther apart as the nanocrystal size decreases (Fig. 5a). Further quantitative results indicate the contractions of $0.7 \%, 1.1 \%$, and $1.4 \%$ relative to the bulk for the 4.0, 2.4, and $1.6 \mathrm{~nm}$ nanocrystals, respectively. The EXAFS data also show a reduction in coordination number and increasing disorder (less intense and broad oscillations).

The normalized $\mathrm{Au} L_{3}$-edge XANES of the three nanocrystals and Au metal (bulk) show some interesting features (Fig. 5b). First, all nanocrystals' spectra exhibit the same line shapes as that of Au metal albeit with a significant broadening, indicating their Au bulk-like environment but with a significant disorder. Second, the white-line intensity increases with the decreasing size of the nanocrystals, suggesting an increase in $d$-hole population $(7.2 \%, 9.0 \%$, and 11.2\% relative to the Au metal) with decreased particle size,

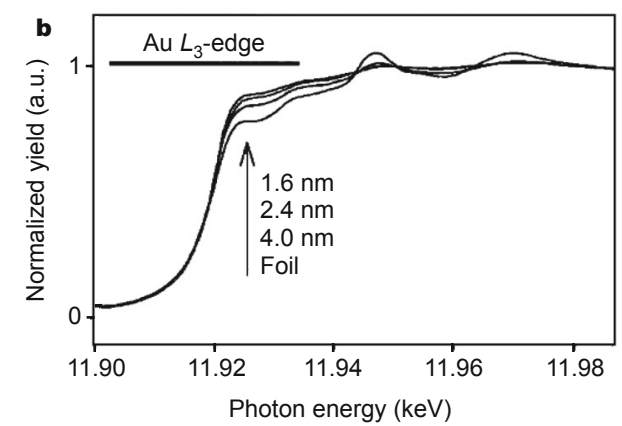

Figure 5 (a) Reverse Fourier transform curves of the nearest $R$-space Au-Au coordination peaks. The inset compares the Fourier transformed $k^{2} \chi(k)$ functions of the $1.6 \mathrm{~nm}$ nanocrystals and Au foil. (b) Au $L_{3}$-edge XANES of the nanocrystals and Au foil. Reprinted with permission from Ref. [50]. Copyright 2003, American Physical Society. 
in good agreement with electronegativity considerations. The implication of these observations is discussed in terms of the interplay of quantum-size and surface effect. It suggests that control over the balance between the size and the nature of the capping molecules could afford some degree of tuning the electronic properties of Au nanocrystals.

Supported metal nanoparticles are widely used as catalysts, whose activity and selectivity are determined by the chemical and structural nature of the catalytically active surface sites. To addresses the structural, dynamic, and electronic features exhibited in a highly prototypical catalytic system, Sanchez and coworkers [51] studied $\gamma-\mathrm{Al}_{2} \mathrm{O}_{3}$ supported Pt nanoparticle catalysts. By collecting EXAFS data for the different-sized (ranging from 0.9 to $2.9 \mathrm{~nm}$ ) samples at the same temperature (Fig. 6a) and the data for a given sample at different temperatures (Fig. 6b), they could discriminate the effects of temperature and particle size on the structural dynamics. The sensitivity to nanoparticle size therefore permits the extraction of quantitative information about particle sizes based on the obtained coordination numbers, which is consistent with the average particle sizes obtained by TEM.

A full comparison of the temperature dependence of the nearest $\mathrm{Pt}-\mathrm{Pt}$ bond lengths for all three samples mea- sured under He (Fig. 6c) reveals that the subnanometer $\mathrm{Pt} / \gamma-\mathrm{Al}_{2} \mathrm{O}_{3}$ sample exhibits a dramatically nonbulk-like nature: it possesses marked relaxations of the $\mathrm{Pt}-\mathrm{Pt}$ bond distances, negative thermal expansion (NTE) with an average linear thermal expansion coefficient of $(-2.4 \pm 0.4)$ $\times 10^{-5} \mathrm{~K}^{-1}$. The temperature-dependence of the EXAFS Debye-Waller factors $\sigma^{2}$ in Fig. $6 \mathrm{~d}$ indicates that the static disorder $\sigma_{\mathrm{s}}^{2}$ and the Einstein temperature increase rapidly as the particle size decreases. Adsorption of hydrogen on the sub-nanometer clusters leads to an increase of the $\mathrm{Pt}-\mathrm{Pt}$ bond lengths and significant attenuation of the disorder. Oxidation of these same clusters has the opposite effect, leading to an increase in $\mathrm{Pt}-\mathrm{Pt}$ bond strain and the subsequent enhancement in nonbulk-like thermal properties. This study highlights that the charge exchange due to both metal-support and metal-adsorbate interactions plays an important role in mediating the structural dynamics of the supported nanoscale metal catalysts. Later, the same group studied the influence of adsorbates on the electronic structure, bond strain of the $\sim 1.0 \mathrm{~nm} \mathrm{Pt} / \gamma-\mathrm{Al}_{2} \mathrm{O}_{3}$ catalyst [52]. They demonstrated that the Pt-Pt bonding in these small clusters is responsive to the presence of adsorbates, exhibiting pronounced coverage-dependent strains, with concomitant modifications of their electronic structures.
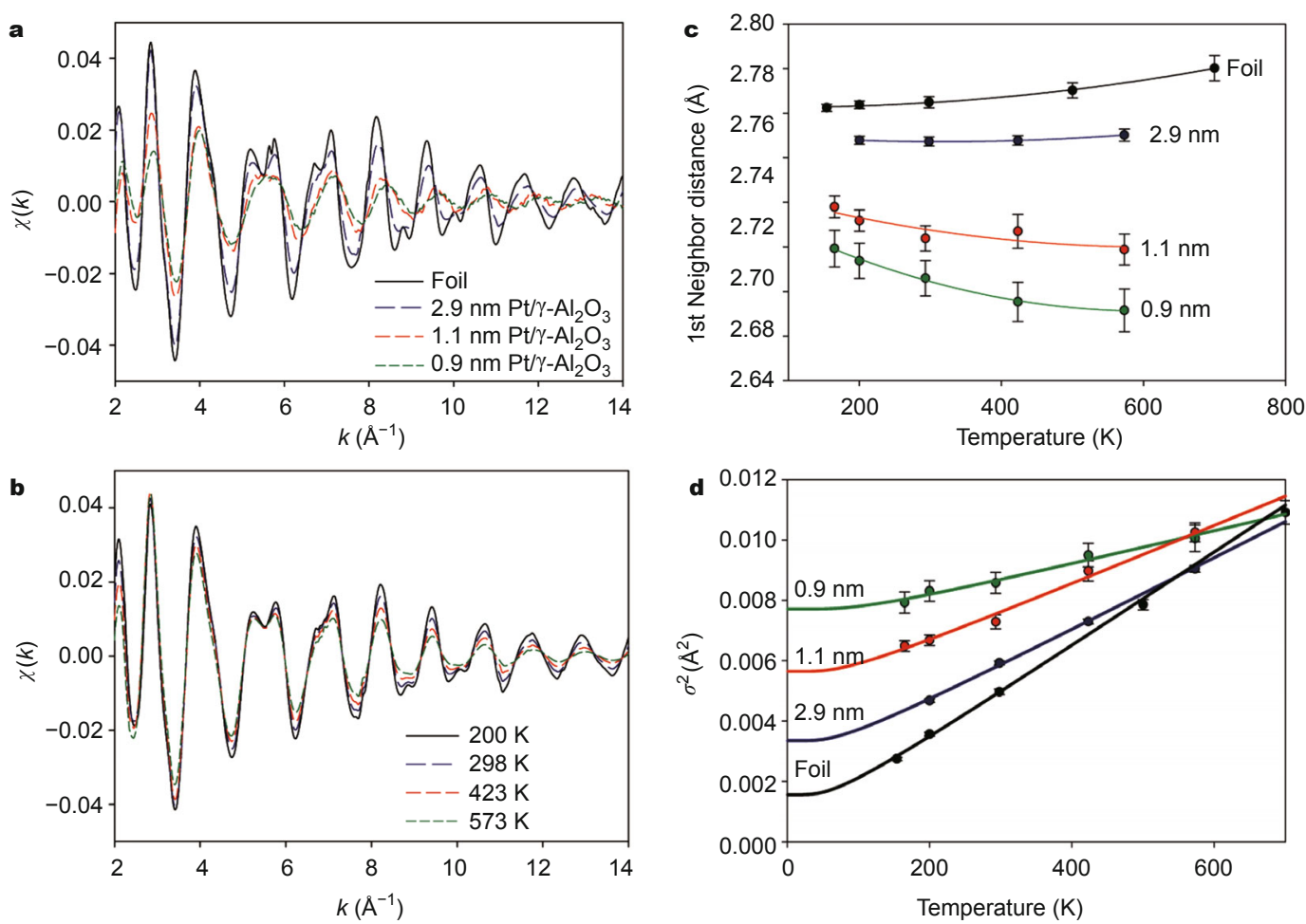

Figure 6 (a) EXAFS $\chi(k)$ functions for different sized $\mathrm{Pt} / \gamma-\mathrm{Al}_{2} \mathrm{O}_{3}$ samples. (b) EXAFS $\chi(k)$ functions for a 2.9 -nm $\mathrm{Pt} / \gamma$ - $\mathrm{Al}_{2} \mathrm{O}_{3}$ sample at different temperatures. (c) Thermal dependence of the Pt-Pt bond lengths for different sized $\mathrm{Pt} / \gamma-\mathrm{Al}_{2} \mathrm{O}_{3}$ nanoparticles, and (d) temperature-dependence of the Debye-Waller factors for the different sized $\mathrm{Pt} / \gamma-\mathrm{Al}_{2} \mathrm{O}_{3}$ nanoparticles, plotted with their respective Einstein models (lines). Reprinted with permission from Ref. [51]. Copyright 2009, American Chemical Society. 
Moreover, the $\mathrm{Pt}-\mathrm{Pt}$ bond strains within the clusters could be relaxed by $\mathrm{H}_{2}$ and $\mathrm{CO}$ adsorbates.

Ligand-protected noble metal nanoclusters ( $\mathrm{Au}, \mathrm{Pt}, \mathrm{Ag}$, $\mathrm{Cu}$, etc.) are a new type of nanomaterials that have been intensely studied in recent years [53-56]. The ultra-small size of these nanoclusters (typically $<2 \mathrm{~nm}$, including tens of metal atoms) make them act as "superatoms" and induce molecular-like properties and distinctive quantum confinement effects, such as discrete electronic structure, highest occupied molecular orbital-lowest unoccupied molecular orbital (HOMO-LUMO) electronic transition, enhanced photoluminescence, and so on. All these unique properties of the nanoclusters are fundamentally different from those of their larger nanocrystals counterparts in which the optical properties are dominated by plasmon excitation and possess a collective nature. The fascinating physicochemical properties of the nanoclusters render them with promising applications in a variety of areas such as photonics, catalysis, sensing, and medicine. To understand the fundamentally important correlation between the electronic structure and the catalytic activity of $\mathrm{Au}_{25}$ clusters, Liu et al. [57] studied two different $\mathrm{Au}_{25}$ clusters, i.e., mixed-ligand (thiol and phosphine) $\left[\mathrm{Au}_{25}\left(\mathrm{PPh}_{3}\right)_{10}\left(\mathrm{SC}_{12} \mathrm{H}_{25}\right)_{5} \mathrm{Cl}_{2}\right]^{2+}$ clusters (called $\mathrm{Au}_{25}$-bi) with bi-icosahedral structure and thiol-stabilized $\left[\mathrm{Au}_{25}\left(\mathrm{SCH}_{2} \mathrm{CH}_{2} \mathrm{Ph}\right)_{18}\right]^{-}$clusters (called $\mathrm{Au}_{25}-\mathrm{i}$ ) with icosahedral core-shell structure. They investigated the catalytic activity of the two clusters for styrene oxidation reaction and correlated their electronic structure information with their catalytic activity by using a combination of XAFS and ultraviolet photoemission spectroscopy (UPS). Compared with bulk Au, the XANES spectra (Fig. 7a) of both $\mathrm{Au}_{25}$ clusters exhibit higher white-line intensity, suggesting the higher $d$-band vacancies in the clusters due to the $\mathrm{Au} \rightarrow \mathrm{S}$ charge transfer. The ligands then not only act as colloidal stabilizers, but also as $d$-band electron acceptor for $\mathrm{Au}$ atoms. Seen from the EXAFS spectra in Fig. 7b, the $\mathrm{Au}_{25}$-bi clusters have a higher first-shell $\mathrm{Au}$ coordination

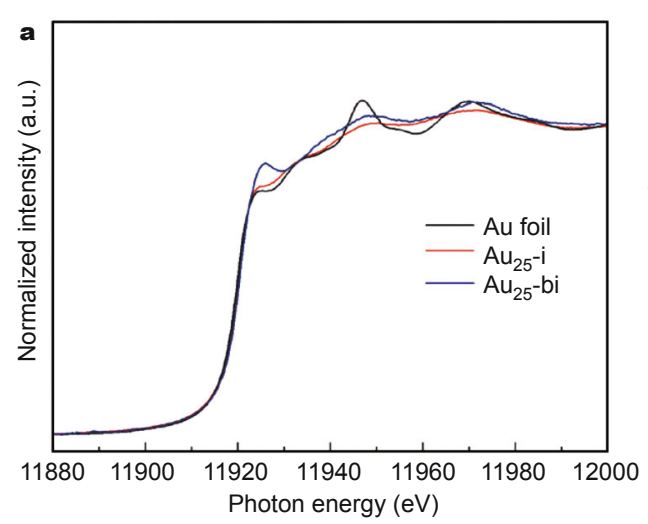

number than $\mathrm{Au}_{25} \mathrm{i}$, in spite of their same number of $\mathrm{Au}$ core atoms in both clusters. The more electropositive nature of clusters resulted in the formation of more oxidative products in the styrene oxidation reaction, thus correlating the electronic and atomic structure of the clusters with catalytic activity.

\section{Oxide nanoparticles}

Hydrogen is a potential solution for satisfying many of our energy needs in the future, and it is mainly produced from the reforming of crude oil, coal, natural gas, wood, organic waste, and biomass. However, $\mathrm{CO}(1-10 \%$ content) present in the reformed fuel degrades the performance of the $\mathrm{Pt}$ electrode used in the fuel cell systems. To produce clean hydrogen, the water-gas shift (WGS, $\mathrm{CO}+\mathrm{H}_{2} \mathrm{O} \rightarrow \mathrm{CO}_{2}$ $+\mathrm{H}_{2}$ ) reaction is a critical industrial process. It is known that metal-oxide nanoparticles are very promising new candidates for high WGS activity, but the design and optimization of these nano-catalysts are hindered by a lack of knowledge about basic questions regarding the nature of the active sites and the reaction mechanism. The nature of the active phases in metal/oxide WGS catalysts and the WGS reaction mechanism are subjects under active debate. To address these issues, in-situ techniques can be quite useful.

Inverse model catalysts of $\mathrm{CeO}_{2}$ nanoparticles supported on $\mathrm{Au}(111)$ or $\mathrm{Cu}(111)$ have shown high catalytic performance in the WGS reaction than the standard $\mathrm{Cu} / \mathrm{CeO}_{2}$ catalysts. Barrio and coauthors [58] used in-situ XRD and $\mathrm{XAFS}$ techniques to monitor the crystalline phases changes and the oxidation states of the $\mathrm{CeO}_{2} / \mathrm{CuO}-\mathrm{Cu}$ catalyst during the WGS reaction, in order to the correlate its structure with activity. Fig. 8a displays the in-situ $\mathrm{Cu} \mathrm{K}$-edge XANES spectra of the $\mathrm{CeO}_{2} / \mathrm{CuO}$ catalyst during the WGS reaction at different temperatures. The XANES spectrum of the starting material demonstrates the features of $\mathrm{CuO}$, and these features are reduced with increasing tempera-

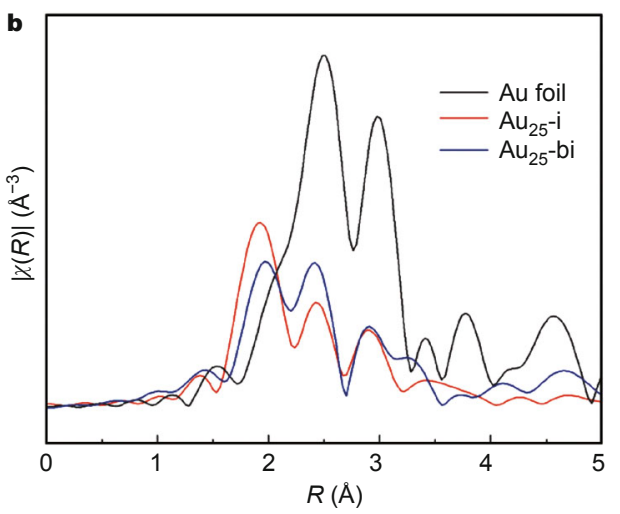

Figure 7 (a) Normalized $\mathrm{Au} L_{3}$-edge XANES spectra of $\mathrm{Au}_{25}$-bi and $\mathrm{Au}_{25}$-i clusters. (b) Fourier transformed EXAFS data of $\mathrm{Au}_{25}$-i and $\mathrm{Au}_{25}$-bi clusters. Reprinted with permission from Ref. [57]. Copyright 2013, Wiley-Blackwell. 
ture. At $200^{\circ} \mathrm{C}$, the spectral profile for $\mathrm{Cu}_{2} \mathrm{O}$ became evident. At $250^{\circ} \mathrm{C}$, the spectral features of XANES matched those of the $\mathrm{Cu}$ bulk, suggesting the complete reduction to $\mathrm{Cu}(0)$ metal in $\mathrm{CeO}_{2} / \mathrm{CuO}$ under WGS reaction conditions. The Ce $L_{3}$-edge XANES was also measured during the WGS reaction as shown in Fig. $8 \mathrm{~b}$ together with spectra for bulk $\mathrm{Ce}^{4+}$ and $\mathrm{Ce}^{3+}$ references. The changes in the spectral features with increasing temperature show that $\mathrm{CeO}_{2}$ was partially reduced under WGS reaction conditions, accompanied by the continuously increased amount of oxygen vacancies and $\mathrm{Ce}^{3+}$ cations. The presence of oxygen vacancies in the ceria lattice is an important property of the active phase of the catalyst, and the catalytic process is likely to occur at the interface of the metallic copper with partially reduced ceria.

Patlolla and cooperators [59] combined XAFS, XRD, and Raman spectroscopy to study the structural and compositional changes of the partially reduced $\mathrm{Fe}_{2} \mathrm{O}_{3}$ and $3 \%$ chromium oxide-modified $\mathrm{Fe}_{2} \mathrm{O}_{3}\left(3 \% \mathrm{Cr}_{2} \mathrm{O}_{3} / \mathrm{Fe}_{2} \mathrm{O}_{3}\right)$ catalysts before, during, and after the WGS reaction. The use of these methods is advantageous since they could provide complementary information on the structure and valence state of $\mathrm{Fe}_{2} \mathrm{O}_{3}$ during reaction. Fig. 9 shows a series of XANES, and EXAFS spectra at Fe $K$-edge collected after exposing $\mathrm{Fe}_{2} \mathrm{O}_{3}$ to $\mathrm{O}_{2}$ at room temperature (RT) and $400^{\circ} \mathrm{C}$, during the WGS reaction at $400^{\circ} \mathrm{C}$ and in the wet gas meter (WGM) flow condition at RT. The XANES spectra show that the $\mathrm{Fe} K$-edge positions measured in $\mathrm{O}_{2}$ flow at RT and $400^{\circ} \mathrm{C}$ are similar, indicating the preservation of the charge state of Fe. However, in WGS conditions at $400^{\circ} \mathrm{C}$, the edge is shifted to the lower energy compared with its oxidized state prior to the WGS reaction, implying the partial reduction of $\mathrm{Fe}$ from $\mathrm{Fe}_{2} \mathrm{O}_{3}$ to $\mathrm{Fe}_{3} \mathrm{O}_{4}$.

The corresponding EXAFS data in Fig. 9b demonstrate significant changes in the local structural environment of Fe in both catalysts, and the changes are markedly different between the two systems. The first nearest $\mathrm{Fe}-\mathrm{O}$ peak located between 1-2.0 A decreases evidently after the WGS reaction. This reduction can be interpreted either by the decreased $\mathrm{Fe}-\mathrm{O}$ coordination number $(\mathrm{CN})$ or the increased bond length disorder or both, but the former factor cannot be too significant due to the similar $\mathrm{Fe}-\mathrm{O}$ coordination numbers in purely octahedrally coordinated $\alpha-\mathrm{Fe}_{2} \mathrm{O}_{3}(\mathrm{CN}$ $=6$ ) to a mixture of two-thirds $\mathrm{Fe}_{2} \mathrm{O}_{3}$ and one-third tetrahedrally coordinated $\mathrm{Fe}_{3} \mathrm{O}_{4}(\mathrm{CN}=5.34)$. The second peak corresponds to the contributions of $\mathrm{Fe}-\mathrm{Fe}$ bonds. Compared with the data under the WGM conditions, the FeFe peak intensity shows a most notable decrease under $\mathrm{O}_{2}$ conditions at the same temperature of $400^{\circ} \mathrm{C}$. Such behavior is consistent with the large disorder in $\mathrm{Fe}$ environment under $\mathrm{O}_{2}$ prior to the onset of the WGS reaction. The interpretation of the EXAFS data is also supported by in situ $\mathrm{XRD}$ results and Raman spectroscopy. For the $3 \% \mathrm{Cr}_{2} \mathrm{O}_{3} /$ $\mathrm{Fe}_{2} \mathrm{O}_{3}$ sample (Fig. 9c), the stronger reduction of the peak intensity compared with the $\mathrm{Fe}_{2} \mathrm{O}_{3}$ sample indicates a more heterogeneous mixture of different $\mathrm{Fe}$ oxide phases with different $\mathrm{Fe}-\mathrm{Fe}$ distances. Combining multiple techniques reveals the role of $\mathrm{Cr}$ in stabilizing the low-temperature $\gamma-\mathrm{Fe}_{2} \mathrm{O}_{3}$ phase, the nature of the disordered phase in the active state of the catalysts, and the possible deactivation mechanism.

\section{Semiconductor quantum structures}

Artificially ordered Ge/Si thin films and superlattices possess exciting electrical and optical properties that open a doorway to band-structure engineering by the formation of strained epitaxial layer of $\mathrm{Si}$ and Ge. A clean $\mathrm{Si}(001)$ surface shows a $(2 \times 1)$ reconstruction and shortened interatomic distance than the counterpart in bulk Si. For Ge overlayers grown on $\mathrm{Si}(001)$, the formation of the dimmer and the interatomic distance are more complicated, depending on the number of $\mathrm{Ge}_{n}$ overlayers. Oyanagi and colleagues [60,61]
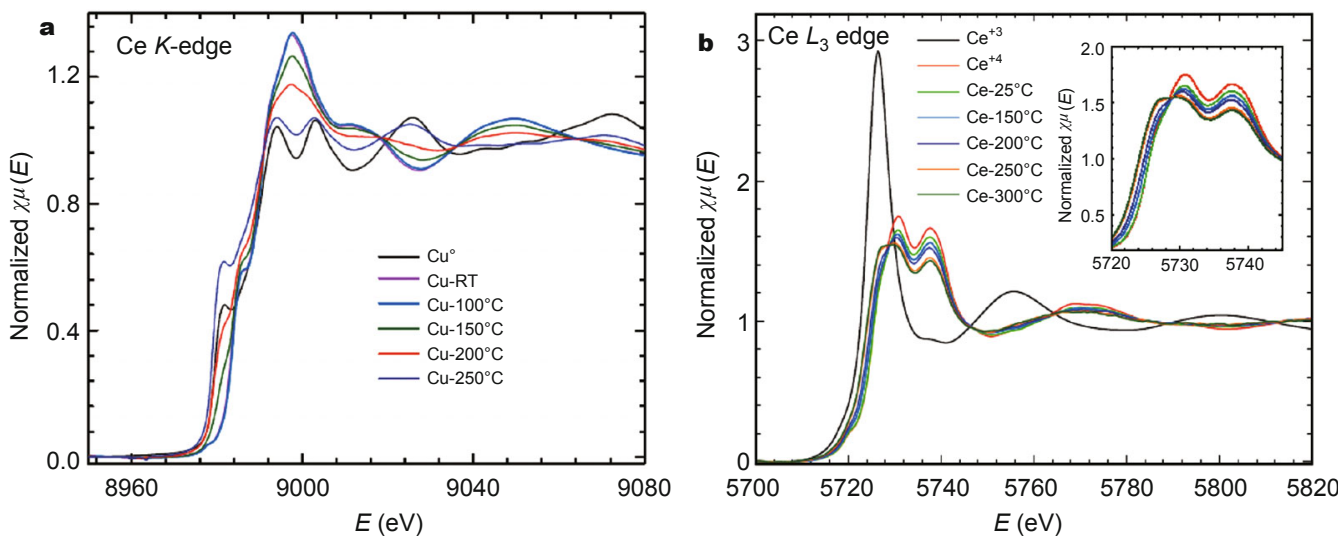

Figure 8 (a) $\mathrm{Cu} K$-edge XANES spectra collected under WGS reaction conditions at various temperatures. (b) Ce $L_{3}$-edge XANES collected under WGS conditions at various temperatures. Reprinted with permission from Ref. [58]. Copyright 2010, American Chemical Society. 

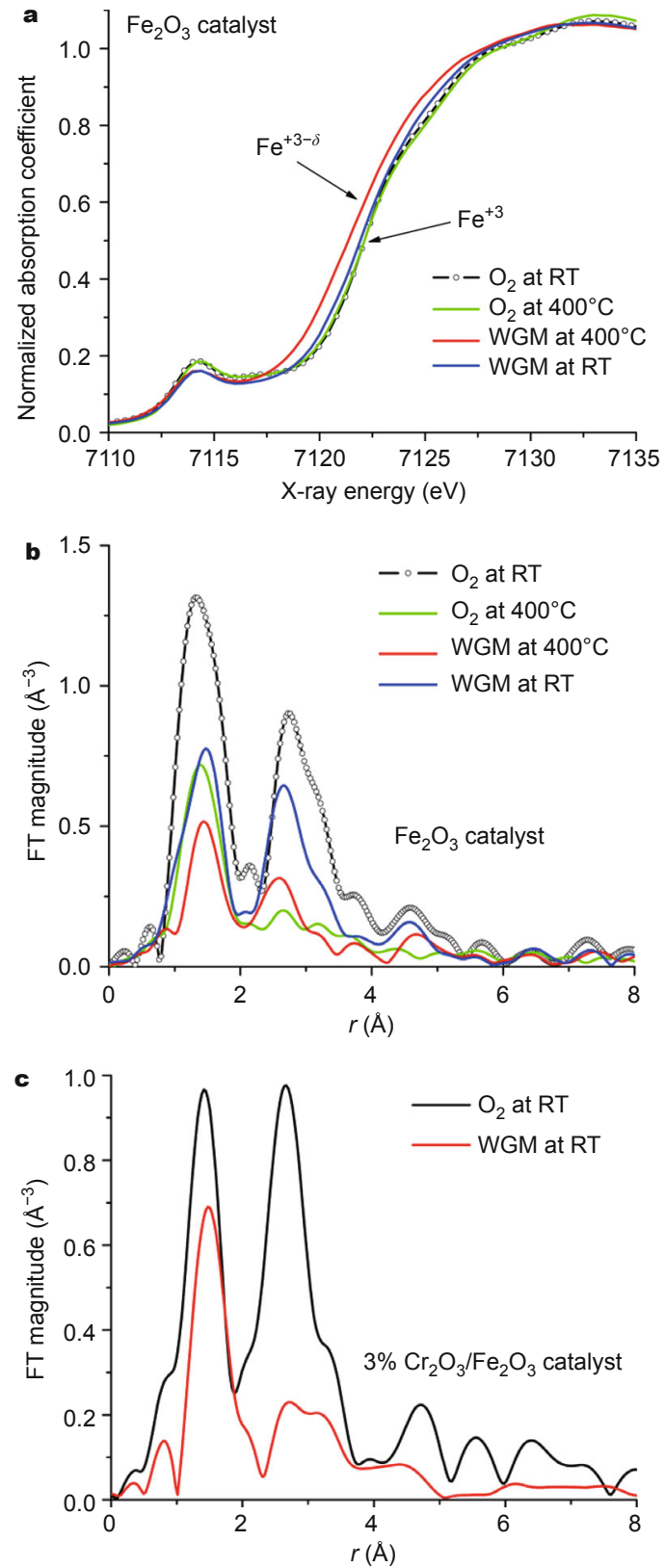

Figure 9 (a) Normalized XANES spectra, and (b) the corresponding EXAFS data at Fe $K$-edge collected after exposing $\mathrm{Fe}_{2} \mathrm{O}_{3}$ to $\mathrm{O}_{2}$ at RT and $400^{\circ} \mathrm{C}$, during the WGS reaction at $400^{\circ} \mathrm{C}$ and in the WGM flow condition at RT. (c) Fe $K$-edge EXAFS spectra for $3 \% \mathrm{Cr}_{2} \mathrm{O}_{3} / \mathrm{Fe}_{2} \mathrm{O}_{3}$ catalysts collected at room temperature in the $\mathrm{O}_{2}$ and WGM flow conditions. Reprinted with permission from Ref. [59]. Copyright 2012, American Chemical Society.

studied the local structure of Ge epitaxial overlayers with Ge thickness from 1 to 4 monolayers (ML) by fluorescence $\mathrm{XAFS}$ in the grazing incidence geometry. The reverse Fourier transformed first-shell Ge $K$-edge EXAFS oscillations are displayed in Fig. 10. The obtained Ge-Ge distance is $2.51 \pm 0.01 \AA$ and Ge-Si distance is $2.40 \pm 0.01 \AA$ for
1-ML Ge on $\mathrm{Si}(001)$, indicating that 1-ML Ge overlayer on $\mathrm{Si}(001)$ forms an elongated dimmer structure. It is interesting that the $\mathrm{Ge}-\mathrm{Ge}$ distance is significantly longer than the interatomic distance $2.45 \AA$ in Ge bulk, in spite of the uniaxial strain due to lattice mismatch which should shorten the bond length within a simple elastic deformation model. These bond length values remain almost unchanged within $\pm 0.01 \AA$ for $2-\mathrm{ML} \mathrm{Ge}$ on $\mathrm{Si}(001)$. However, upon increasing the number of $\mathrm{Ge}_{n}$ overlayers to $n=4$, the $\mathrm{Ge}-\mathrm{Ge}$ distance is reduced to $2.47 \pm 0.01 \AA$ and the Ge-Si distance is $2.38 \pm$ $0.01 \AA$. Fig. 10b shows the surface strain in 1-ML and 2-ML Ge on $\mathrm{Si}(001)$ schematically. Stress components are indicated by the directions of arrows. Strains are induced by the surface reconstruction (dimmer strain) and the atomic size effect (mismatch strain). A possible channel of atomic migrations is indicated by arrows in the bottom figure.

A $\left(\mathrm{Ge}_{4} \mathrm{Si}_{4}\right)_{5}$ monolayer strained-layer superlattice grown on $\mathrm{Si}(001)$ substrate shows strong optical transitions at $0.75,1.25$, and $2.31 \mathrm{eV}$ unique to the superlattice period, found neither in constituent crystals nor in the $\mathrm{Ge}_{0.5} \mathrm{Si}_{0.5}$ alloy [62]. Using grazing-incidence fluorescence EXAFS, Wei and colleagues [63] studied the local structure of $\left(\mathrm{Ge}_{4}\right.$ $\left.\mathrm{Si}_{4}\right)_{5}$ strained-layer superlattice. EXAFS spectra for both $\left(\mathrm{Ge}_{4} \mathrm{Si}_{4}\right)_{5}$ and $\mathrm{Ge}_{0.5} \mathrm{Si}_{0.5} / \mathrm{Si}(001)$ have an intermediate profile, suggesting that these two samples are characterized by an almost equal amount of Ge and Si scatterers (Fig. 11a). The least-squares curve-fitting results indicate that the bond lengths for $\left(\mathrm{Ge}_{4} \mathrm{Si}_{4}\right)_{5}$ are $R_{\mathrm{Ge}-\mathrm{Ge}}=2.42 \pm 0.01 \AA, R_{\mathrm{Ge}-\mathrm{Si}}=2.38$ $\pm 0.01 \AA$, and the coordination numbers are $N_{\mathrm{Ge}}=1.8$ and $N_{\mathrm{Si}}=2.2$, respectively. $R_{\mathrm{Ge}-\mathrm{Si}}=2.38 \AA$ in $\left(\mathrm{Ge}_{4} \mathrm{Si}_{4}\right)_{5}$ is identical with the value for the dilute Ge atoms doped into Si crystal, suggesting that the bond-length compression takes the upper limit of the isotropic deformation. The observed $R_{\mathrm{Ge}-\mathrm{Ge}}$ and $R_{\mathrm{Ge}-\mathrm{Si}}$ values take slightly larger values than the calculated values for the model $\left(\mathrm{Ge}_{4} \mathrm{Si}_{4}\right)_{5}$ superlattice. The overestimation of bond-length compression may occur since most of the model structures do not take interface mixing and non-uniform longitudinal displacement into account. Based on these results, it may be concluded that the lattice matching is achieved by tetragonal deformation via bond bending but the Ge layer is highly compressed. Fig. 11b illustrates the schematic structures for $\left(\mathrm{Ge}_{4} \mathrm{Si}_{4}\right)_{5}$ with an ideal $\mathrm{Ge} / \mathrm{Si}$ interface and with an interface mixing. In the latter model, the Ge overlayer is assumed to have $0.5 \mathrm{ML}$ site exchange upon the deposition and $1 \mathrm{ML}$ segregation upon $\mathrm{Si}$ overlayer growth.

The self-assembled Ge/Si quantum dots (QDs) is also an interesting research topic. Although the Stranski-Krastanov (S-K) mode is commonly used to describe the growth of $\mathrm{Ge} \mathrm{QD}$ on $\mathrm{Si}$, due to the intermixing between $\mathrm{Ge}$ and $\mathrm{Si}$, the real formation of Ge QDs is much more complicated. Moreover, the capping of the Ge islands by $\mathrm{Si}$, which is 

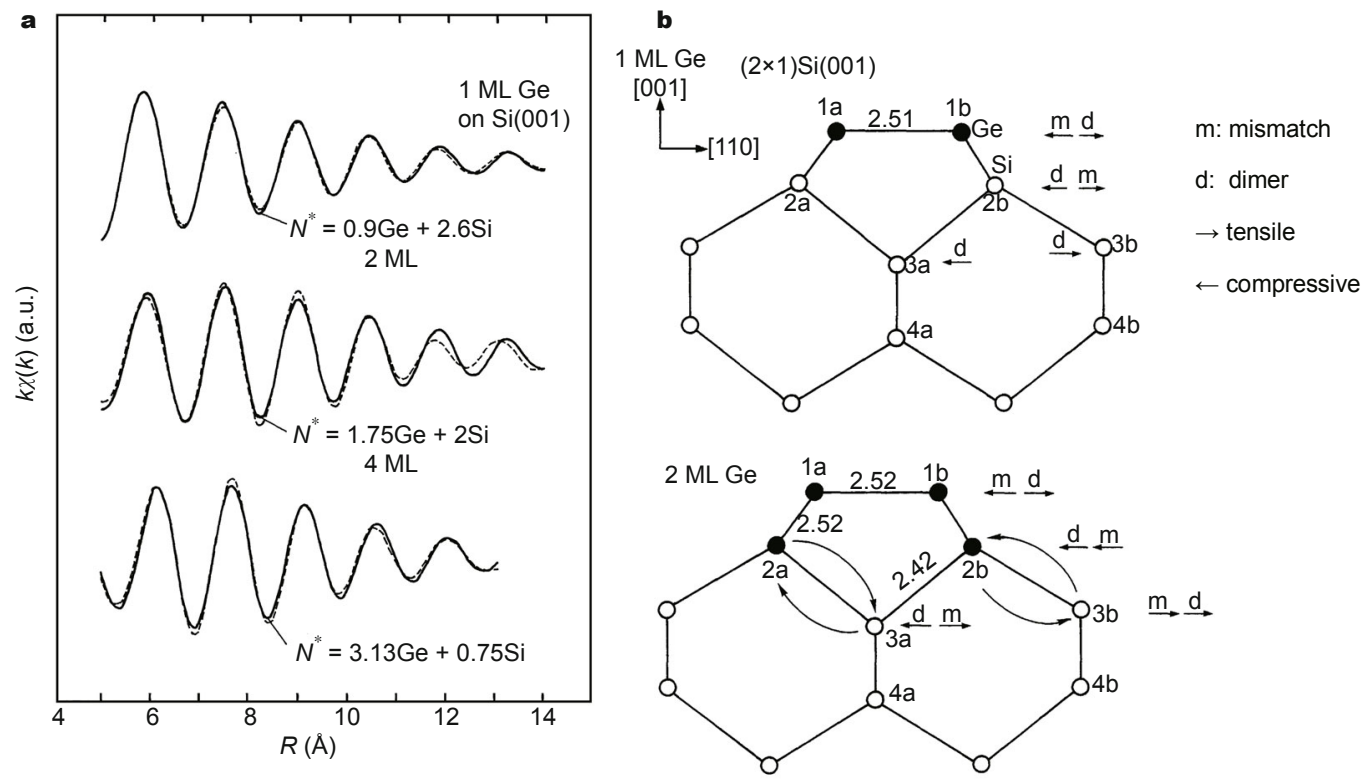

Figure 10 (a) The first-shell EXAFS oscillations and the calculated curves of model structure for 1-4-ML Ge on $\mathrm{Si}(001)$. (b) The schematic surface strain in 1- and 2-ML Ge on $\mathrm{Si}(001)$. Reprinted with permission from Ref. [60]. Copyright 1994, the Japan Society of Applied Physics.
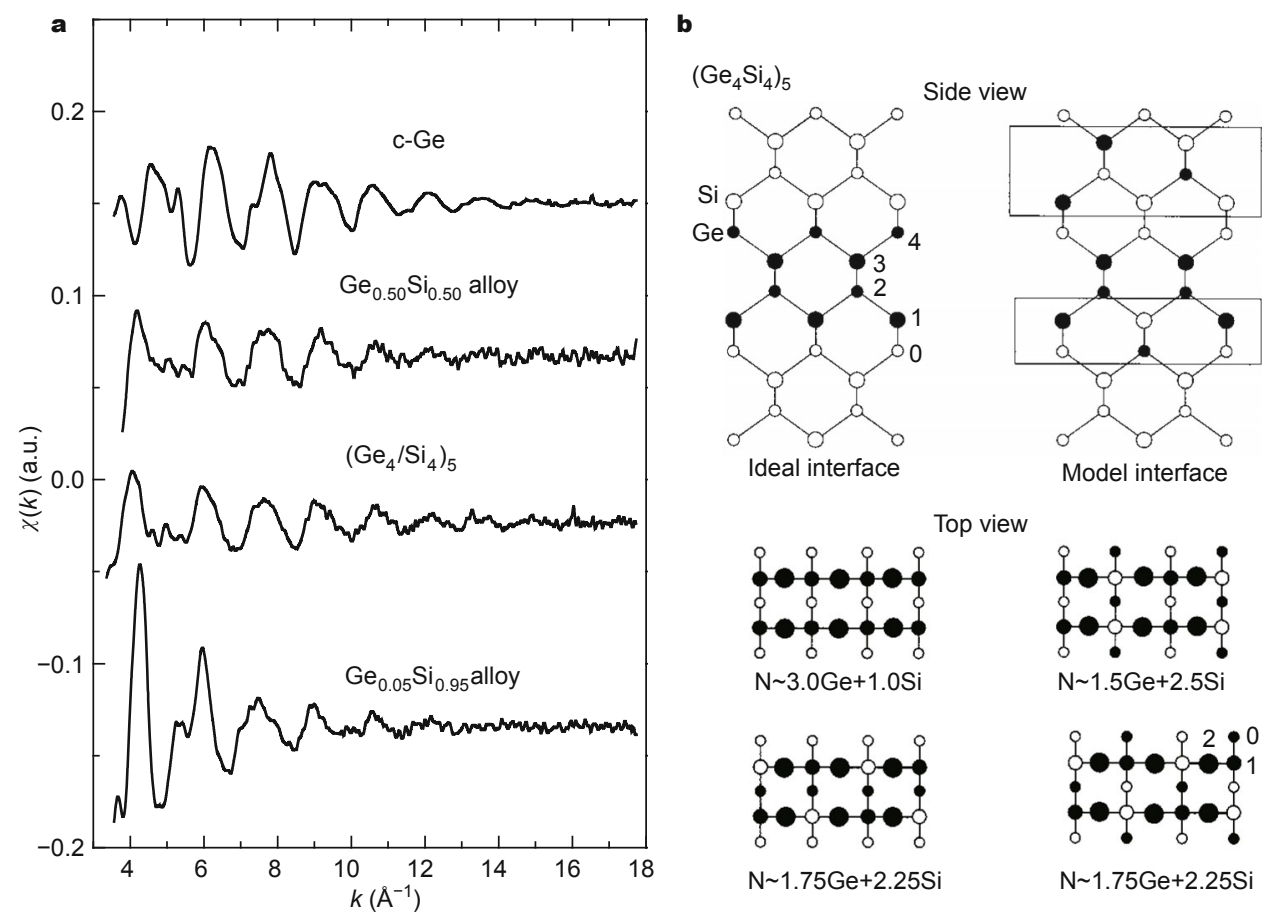

Figure 11 (a) The EXAFS $\chi(k)$ function for $\left(\mathrm{Ge}_{4} \mathrm{Si}_{4}\right)_{5}$, crystalline $\mathrm{Ge}$ (c-Ge) and $\mathrm{Ge}_{x} \mathrm{Si}_{1-x}$ alloys. (b) The schematic structures for $\left(\mathrm{Ge}_{4} \mathrm{Si}_{4}\right)_{5}$ with an ideal Ge/Si interface (left) and with an interface mixing (right). Reprinted with permission from Ref. [63]. Copyright 2000, American Physical Society.

necessary to avoid oxidation, is also likely to modify the morphology, structure, electronic and optical properties of $\mathrm{Ge}$ QDs [64]. A simultaneous determination of their strain and composition is of interest. Sun et al. [65] first used the EXAFS method to study the local structures around $\mathrm{Ge}$ atoms in the first three coordination shells for self-assembled the Ge QDs grown at $510^{\circ} \mathrm{C}$ and then capped by $\mathrm{Si}$ at different temperatures $\left(300\right.$ and $\left.510^{\circ} \mathrm{C}\right)$. The authors first performed a preliminary multiple-scattering fitting on the data of crystalline Ge using all 10 scattering paths in the 
first three shells. It is found that the experimental EXAFS data for the diamond structure can be well reproduced by considering only the single-scattering paths plus one dominant multiple-scattering (MS) path $\mathrm{Ge}_{0} \rightarrow \mathrm{B}_{1} \rightarrow \mathrm{B}_{2} \rightarrow \mathrm{Ge}_{0}$. Based on the structure model of the Ge QDs (inset of Fig. 12), EXAFS contributions from the environments around $\mathrm{Ge}$ in the islands and the wetting layer were separated. The fitting was done in $R$-space for the first three shells as shown in Fig. 12. From the coordination numbers and considering the interface effect, the average Ge concentrations in the islands were estimated to be $\sim 0.9$ and $\sim 0.4$ for the QDs capped at 300 and $510^{\circ} \mathrm{C}$, respectively, demonstrating much stronger $\mathrm{Ge} / \mathrm{Si}$ intermixing at the capping temperature of $510^{\circ} \mathrm{C}$ than at $300^{\circ} \mathrm{C}$. For the Ge QDs capped by $\mathrm{Si}$ at $300^{\circ} \mathrm{C}$, from the first shell bond lengths $R_{\mathrm{Ge}-\mathrm{Ge}}(2.43$ $\AA$ ) and $R_{\mathrm{Ge}-\mathrm{Si}}(2.37 \AA)$ as well as the estimated in-plane and out-of-plane bond angle distortion $\Delta \theta\left(-2.0^{\circ}\right)$ and $\Delta \theta^{\prime}\left(1.0^{\circ}\right)$ calculated by the elastic theory, the local strain in the nearest neighbor around $\mathrm{Ge}$ is mainly accommodated by the bond bending.

\section{Magnetic semiconductor nanostructures}

The rapidly developing field of spintronics requires the simultaneous manipulation of spin and charge $[66,67]$, which

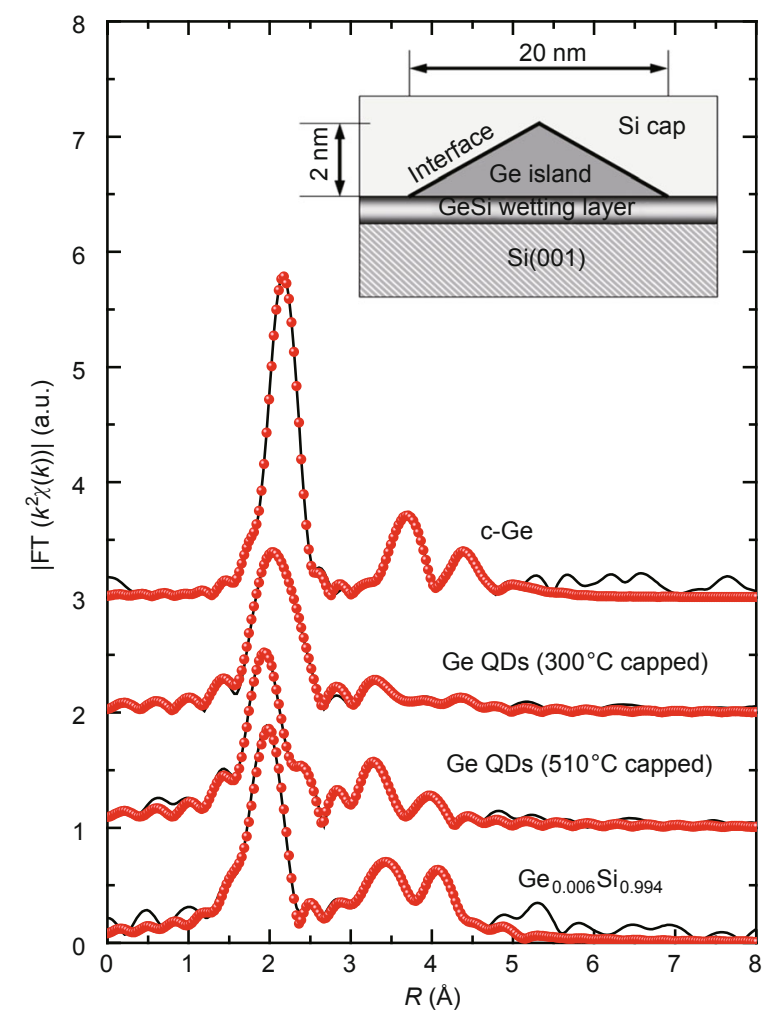

Figure 12 The Fourier transformed $k^{2} \chi(k)$ functions for Ge QDs grown at $510^{\circ} \mathrm{C}$ and capped by $\mathrm{Si}$ at different temperatures: experiment (solid) and fit (open circle). The inset shows the schematic cross-sectional geometry of the Ge QDs. could provide enhanced functionality and performance in traditional charge-based electronics. One of the promising material candidates for the semiconductor spintronics is diluted magnetic semiconductor (DMS) which refer to semiconductor substitutionally incorporated by transition-metal (TM) magnetic ions [68]. A magnetically doped semiconductor could be called a "true" DMS material only when the dopants occupy the substitutional positions in the host, as suggested by the prediction of Dietl et al. [69]. In reality, the doped TM ions could occupy different positions (substitutional sites, interstitials) in the host lattice, or form metallic clusters/ secondary phases [70], all of which exert impacts to the magnetic properties of the DMSs. Therefore, structural studies play an extremely important role in understanding the magnetic nature in DMSs. However, identifying the occupation sites of the TM dopants in DMSs is not a trivial task as there are very few techniques capable of solving this issue. Due to its sensitivity to the local atomic/ electronic structures and the element-specificity, XAFS has proved to be a unique technique in identifying the occupation sites and the distribution of the dopants, detecting the presence of metal clusters or secondary phases, as well as identifying the defect types and the dopant valence.

XANES has made a significant contribution to the identification of the occupation sites of TM dopants in DMS materials. Wei and colleagues [71] investigated an Mndoped GaN system by comparison of experimental and theoretical spectra of XANES. Theoretical XANES spectra of the representative structure models of $\mathrm{Mn}$ in GaN lattice were calculated, and compared with the experimental data of 300-nm thick $\mathrm{Ga}_{1-x} \mathrm{Mn}_{x} \mathrm{~N}(x=0.010,0.025,0.10)$ thin films grown on $\mathrm{GaAs}(001)$ substrates using plasma-assisted molecular beam epitaxy (Fig. 13a) [72]. It is shown that the substitutional $\mathrm{Mn}\left(\mathrm{Mn}_{\mathrm{Ga}}\right)$ in $\mathrm{GaN}$ is characterized by a pre-edge peak at $2.0 \mathrm{eV}$ and a post-edge multiple-scattering peak at $29.1 \mathrm{eV}$. The peaks are shifted in position and dropped in intensity dramatically for the interstitial $\mathrm{Mn}_{\mathrm{I}}$ and $\mathrm{Mn}_{\mathrm{Ga}}-\mathrm{Mn}_{\mathrm{I}}$ dimer, and then disappear completely for the metallic Mn clusters. The comparison between the experimental and theoretical XANES spectra indicate that the majority of $\mathrm{Mn}$ atoms locate at the $\mathrm{Mn}_{\mathrm{Ga}}$ sites in $\mathrm{Ga}_{1-x}$ $\mathrm{Mn}_{x} \mathrm{~N}$ with the lowest $\mathrm{Mn}$ content (0.010). Upon increasing the Mn content to 0.025 , a significant fraction of $\mathrm{Mn}$ dopants exist in the form of $\mathrm{Mn}_{\mathrm{Ga}}-\mathrm{Mn}_{\mathrm{I}}$ dimers. At the highest Mn doping concentration (0.100), a significant amount of metallic Mn clusters were precipitated. Kunisu et al. [73] also reported a Mn K-edge XANES study on the local environment of $\mathrm{Mn}$ dopants in $\mathrm{ZnO}$ polycrystals. The spectra as shown in Fig. 13b at the Mn concentrations between 0.01 and 5 at.\% indicate almost identical features, which could be well reproduced by first-principles spin-polarized density functional theory (DFT) calculations for substitu- 


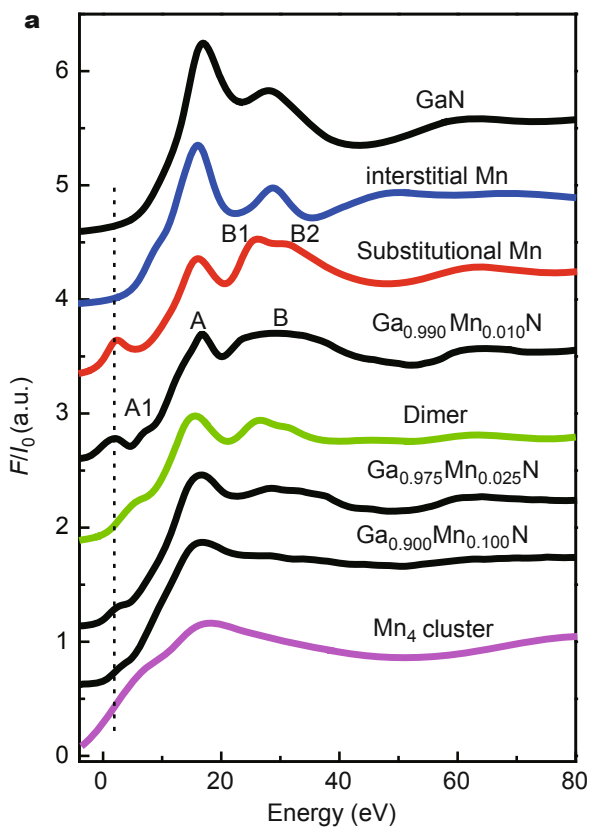

Figure 13 (a) Comparison of the experimental XANES spectra for $\mathrm{Ga}_{1-x} \mathrm{Mn}_{x} \mathrm{~N}$ with various $M n$ concentration with the calculated Mn $K$-edge XANES spectra for representative structure models. Reprinted with permission from Ref. [71]. Copyright 2006, American Institute of Physics. (b) XANES spectra at $\mathrm{Mn} K$-edge for polycrystalline Mn-doped $\mathrm{ZnO}$ polycrystals with $\mathrm{Mn}$ concentrations of $0.01,5,10$, and 20 at.\%. Reprinted with permission from Ref. [73]. Copyright 2005, American Institute of Physics. tional $\mathrm{Mn}$ at $\mathrm{Zn}$ sites in $\mathrm{ZnO}$. The XANES at 10 at.\% Mn is also very similar to the ones with lower concentrations, whereas a minor portion of Mn exist in the form of $\mathrm{Mn}_{3} \mathrm{O}_{4}$ as found in XRD pattern. At a higher Mn concentration of 20 at.\%, change in the XANES shape is clearly recognized.

Sun and coworkers [74] also combined the Co and O $K$-edges spectra to investigate the Co occupation sites and distributions in $\mathrm{Zn}_{1-x} \mathrm{Co}_{x} \mathrm{O}$ thin films with low Co concentrations $(x=0.005,0.02)$. Co $K$-edge EXAFS analysis indicates unambiguously that the doped Co ions are substantially incorporated into the $\mathrm{ZnO}$ host lattice. However, strikingly different features in the $\mathrm{O} K$-edge XANES features for the $\mathrm{Zn}_{1-x} \mathrm{Co}_{x} \mathrm{O}$ films than those for $\mathrm{ZnO}$ bulk are observed, which can be reproduced by the assembling of the substitutional Co ions via intervening oxygen atoms. This provides experimental evidence for the theoretical predications $[75,76]$ that the substitutional Co ions in $\mathrm{ZnO}$ tend to gather together, rather than distribute randomly in the whole lattice.

Interests in the Co-doped $\mathrm{ZnO}$ system have been extended to zero-dimensional and one-dimensional nanostructures. Especially, Co-doped $\mathrm{ZnO}$ nanowires (NWs) have attracted considerable attention. Yuhas and colleagues [77] synthesized single crystalline, single domain, and single phase Co:ZnO NWs with uniform diameters of $35 \pm$ $5 \mathrm{~nm}$ and lengths ranging from 2 to $6 \mu \mathrm{m}$ using a solution-based synthetic route. The Co concentrations, ranging from 5.35 to 15.50 at.\%, are far beyond the equilibrium solubility of Co in bulk $\mathrm{ZnO}$. Whereas, the doped Co ions reside in a uniform subsitutional environment with a small degree of disorder. A similar phenomenon was also observed by Yao and coworkers on the $\mathrm{Zn}_{0.98} \mathrm{Co}_{0.02} \mathrm{O} \mathrm{NWs}$ [78]. The substitutional incorporation of $\mathrm{Mn}$ ions up to $10 \%$ into $\mathrm{CdSe}$ quantum nano-ribbons was also readily achieved by $\mathrm{Yu}$ and coworkers via a nucleation-controlled doping process [79].

Segura-Ruiz and coworkers [80] used a hard X-ray nanoprobe to investigate the local structure of single Coimplanted $\mathrm{ZnO} \mathrm{NWs}$ with a Co concentration of 0.6 at.\% relative to $\mathrm{Zn}$. The monochromatic $\mathrm{X}$-ray beam was focused to $100 \mathrm{~nm} \times 100 \mathrm{~nm}$ spot size with $\sim 10^{11} \mathrm{ph} / \mathrm{s}$ using a pair of Kirkpatrick-Baez Si mirrors. The uniform $\mathrm{Zn}$ and Co distributions along the wires are confirmed by X-ray fluorescence maps. EXAFS analysis reveals the substitution of $\mathrm{Co}^{2+}$ ions in $\mathrm{Zn}$ sites and the high structural order in the host lattice. The polarization-dependent $\mathrm{Zn} \mathrm{K}$-edge XANES spectra at different points along the NW shown in Fig. 14 reveal the high structural order of the host lattice along both radial and axial directions. Specifically, the XANES data with the electric field of the absorbed photons perpendicular to the $c$-axis $(E \perp c)$ probe the symmetry of the $a b$-plane, namely $p_{x y}$ conduction band states. The spectral features in Fig. 14a reflect the typical wurtzite structure of the $\mathrm{ZnO}$ lattice, and the signatures of $\mathrm{Zn}$ metallic precipitates after the ion implantation process are observed. No structural disorder in the radial direction along the implanted NW is suggested according to the line shapes and the peaks energies. For the axial direction $(E / / c)$, the XANES data probe the symmetry of the $c$-plane, namely $p_{z}$ conduction band states. Again, the spectra exhibit the 


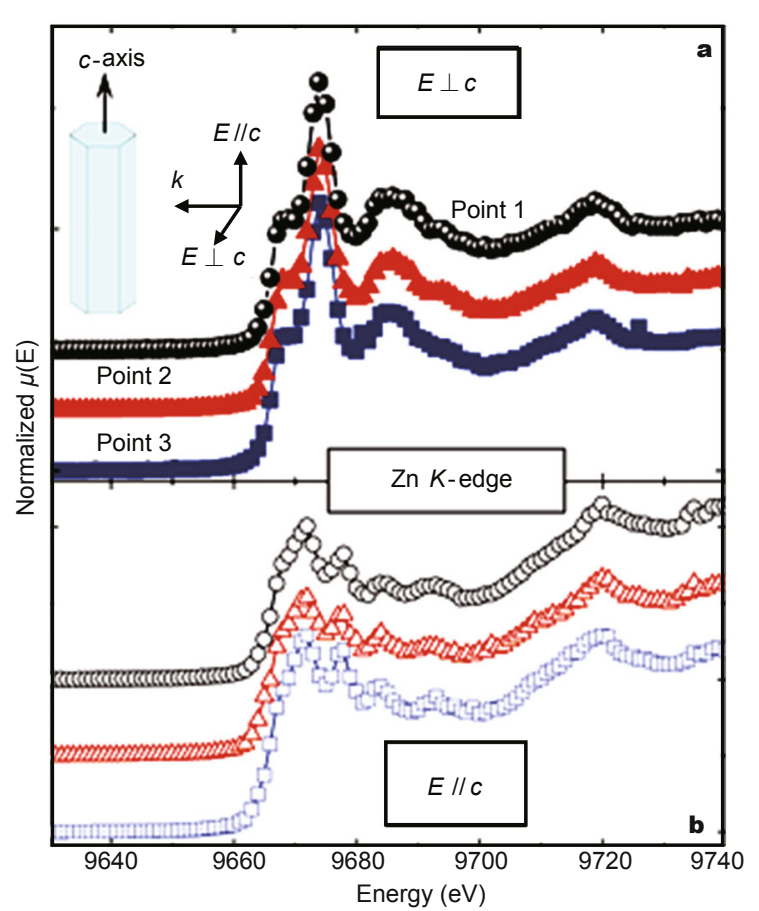

Figure 14 Polarization-dependent $\mathrm{Zn} K$-edge XANES spectra of Coimplanted ZnO NWs: (a) $\boldsymbol{E} \perp \boldsymbol{c}$, and (b) $\boldsymbol{E} / / \boldsymbol{c}$. Reprinted with permission from Ref. [80]. Copyright 2011, American Chemical Society.

peaks associated to the hexagonal structure, without any evidence of lattice damage along the NW (Fig. 14b).

\section{Core/shell and hybrid nanostructures}

In diluted magnetic semiconductor quantum dots (DMSQDs), the quantum confinement suppresses the ferromagnetic couplings between the doped magnetic ions. In this size range, introducing extra charge carriers as routinely used for bulk DMSs is not an ideal pathway. To realize the ferromagnetic coupling between magnetic ions in DMSQDs still remains rather challenging. Yan and coauthors [81] proposed that core/shell structure might provide an effective approach to tune ferromagnetic exchange in DMSQDs, because it engineers the energy level of the magnetic impurity $3 d$ levels relative to the band edge. This idea has been successfully applied to $\mathrm{Zn}_{0.96} \mathrm{Co}_{0.04} \mathrm{O}$ DMSQDs covered by a shell of $\mathrm{ZnS}$ or $\mathrm{Ag}_{2} \mathrm{~S}$.

Experimentally, by the growth of a shell of $\mathrm{ZnS}$ or $\mathrm{Ag}_{2} \mathrm{~S}$ around the 5-nm sized Co-doped $\mathrm{ZnO}$ QDs cores in an epitaxial-like manner, $\mathrm{Zn}_{0.96} \mathrm{Co}_{0.04} \mathrm{O} / \mathrm{ZnS}$ (type-II core/ shell) and $\mathrm{Zn}_{0.96} \mathrm{Co}_{0.04} \mathrm{O} / \mathrm{Ag}_{2} \mathrm{~S}$ (inverted core/shell) core/ shell structures were synthesized, which were demonstrated to be able to turn the magnetic couplings within the Codoped $\mathrm{ZnO}$ QDs from antiferromagnetic into ferromagnetic. Magnetization measurement results show that the growth of a $\mathrm{ZnS}$ shell turns the $\mathrm{Co}^{2+}-\mathrm{Co}^{2+}$ magnetic cou- plings in $\mathrm{Zn}_{0.96} \mathrm{Co}_{0.04} \mathrm{O}$ QDs from antiferromagnetic into ferromagnetic, with a saturation moment as high as $2.8 \mu_{\mathrm{B}} /$ $\mathrm{Co}^{2+}$. The $\mathrm{Zn} \mathrm{K}$-edge XANES spectrum of $\mathrm{Zn}_{0.96} \mathrm{Co}_{0.04} \mathrm{O} /$ $\mathrm{ZnS}$ exhibits a similar spectral shape to that of $\mathrm{Zn}_{0.96} \mathrm{Co}_{0.04} \mathrm{O}$ QDs (Fig. 15a), although the white-line peak at $\sim 9673 \mathrm{eV}$ is significantly reduced in intensity (Fig. 15b). They considered several structure models and calculated the $\mathrm{Zn} \mathrm{K}$-edge XANES spectra (Fig. 15c). The calculation results suggest that $\mathrm{ZnO} / \mathrm{ZnS}$ core/shell structure model could reproduce the main spectral features of the experimental data for $\mathrm{Zn}_{0.96} \mathrm{Co}_{0.04} \mathrm{O} / \mathrm{ZnS}$, providing support for the formation of a $\mathrm{ZnS}$ layer on the $\mathrm{Zn}_{0.96} \mathrm{Co}_{0.04} \mathrm{O}$ QDs surfaces. From the $\mathrm{ZnS} / \mathrm{ZnO}$ molar ratio (40:60) obtained from the quantitative EXAFS data analysis (Fig. 15d) and considering the particle size $(\sim 5 \mathrm{~nm})$, a $\mathrm{ZnS}$ shell thickness of about 0.55 $\mathrm{nm}$ was estimated. Detailed analysis of EXAFS spectra in Fig. $15 \mathrm{e}$ reveals the substitution of Co for $\mathrm{Zn}$ sites in all these QDs, ruling out the possibility of forming any Co-related secondary phases. Based on these structure study results, the significantly enhanced saturation magnetic moment of $\mathrm{Zn}_{0.96} \mathrm{Co}_{0.04} \mathrm{O} / \mathrm{ZnS}$ (and $\mathrm{Zn}_{0.96} \mathrm{Co}_{0.04} \mathrm{O} / \mathrm{Ag}_{2} \mathrm{~S}$ ) QDs is proposed to arise from the modified exchange interactions between the substitutional Co ions.

The same group also used hybrid structure of $\mathrm{Zn}_{0.98}$ $\mathrm{Co}_{0.02} \mathrm{O}$ QDs and reduced graphene oxide (RGO) to tune the magnetic properties of DMSQDs [82]. They synthesized three distinct samples, namely, $\mathrm{Zn}_{0.98} \mathrm{Co}_{0.02} \mathrm{O}, \mathrm{Zn}_{0.98^{-}}$ $\mathrm{Co}_{0.02} \mathrm{O}$-RGO and $\mathrm{Zn}_{0.98} \mathrm{Co}_{0.02} \mathrm{O} / \mathrm{RGO}$ QDs. $\mathrm{Zn}_{0.98} \mathrm{Co}_{0.02} \mathrm{O} /$ RGO refers to a quasi-core/shell structure in which the $\mathrm{Zn}_{0.98} \mathrm{Co}_{0.02} \mathrm{O}$ cores are almost fully covered by RGO shells, and $\mathrm{Zn}_{0.98} \mathrm{Co}_{0.02} \mathrm{O}$-RGO means $\mathrm{Zn}_{0.98} \mathrm{Co}_{0.02} \mathrm{O}$ QDs anchored on RGO sheets but not completely wrapped. Magnetization measurements show that after covering the RGO shell, the $\mathrm{Zn}_{0.98} \mathrm{Co}_{0.02} \mathrm{O} / \mathrm{RGO}$ QDs display a well-defined hysteresis loop, exhibiting a room-temperature ferromagnetic behavior. A similar phenomenon is also observed in $\mathrm{Zn}_{0.98} \mathrm{Co}_{0.02} \mathrm{O}-\mathrm{RGO}$, but its saturation magnetic moment $\left(0.1 \mu_{\mathrm{B}} / \mathrm{Co}\right)$ is evidently smaller than that $\left(0.3 \mu_{\mathrm{B}} / \mathrm{Co}\right)$ of $\mathrm{Zn}_{0.98} \mathrm{Co}_{0.02} \mathrm{O} / \mathrm{RGO}$. Fig. 16a displays the Fourier transform of Co K-edge EXAFS $k^{3} \chi(k)$ functions for $\mathrm{Zn}_{0.98} \mathrm{Co}_{0.02} \mathrm{O}$, $\mathrm{Zn}_{0.98} \mathrm{Co}_{0.02} \mathrm{O}$-RGO and $\mathrm{Zn}_{0.98} \mathrm{Co}_{0.02} \mathrm{O} / \mathrm{RGO}$, which rule out the existence of Co metal and cobalt oxides (like $\mathrm{CoO}$ and $\left.\mathrm{Co}_{3} \mathrm{O}_{4}\right)$ in the samples. $\mathrm{C} K$-edge XANES measurements (Fig. 16b) show characteristic peaks of C $1 s \rightarrow \pi^{*}(\sim 285.5 \mathrm{eV})$ and $\mathrm{C} 1 s \rightarrow \sigma^{*}(\sim 292.5 \mathrm{eV})$ transitions for RGO. Compared with $\mathrm{RGO}, \mathrm{Zn}_{0.98} \mathrm{Co}_{0.02} \mathrm{O} / \mathrm{RGO}$ hybrid shows a clearly enhanced intensity of the peak at $\sim 288 \mathrm{eV}$, most possibly due to the binding of oxygen or other species to the carbon atoms in RGO, suggesting the formation of the interfacial $\mathrm{Zn}(\mathrm{Co})-\mathrm{O}-\mathrm{C}$ bonds (inset of Fig. 16b) in the $\mathrm{Zn}_{0.98} \mathrm{Co}_{0.02} \mathrm{O}$ / RGO hybrid. The Co $L_{2,3}$-edge XANES spectra of $\mathrm{Zn}_{0.98^{-}}$ $\mathrm{Co}_{0.02} \mathrm{O}$ and $\mathrm{Zn}_{0.98} \mathrm{Co}_{0.02} \mathrm{O} / \mathrm{RGO}$ as displayed in Fig. $16 \mathrm{c}$ 

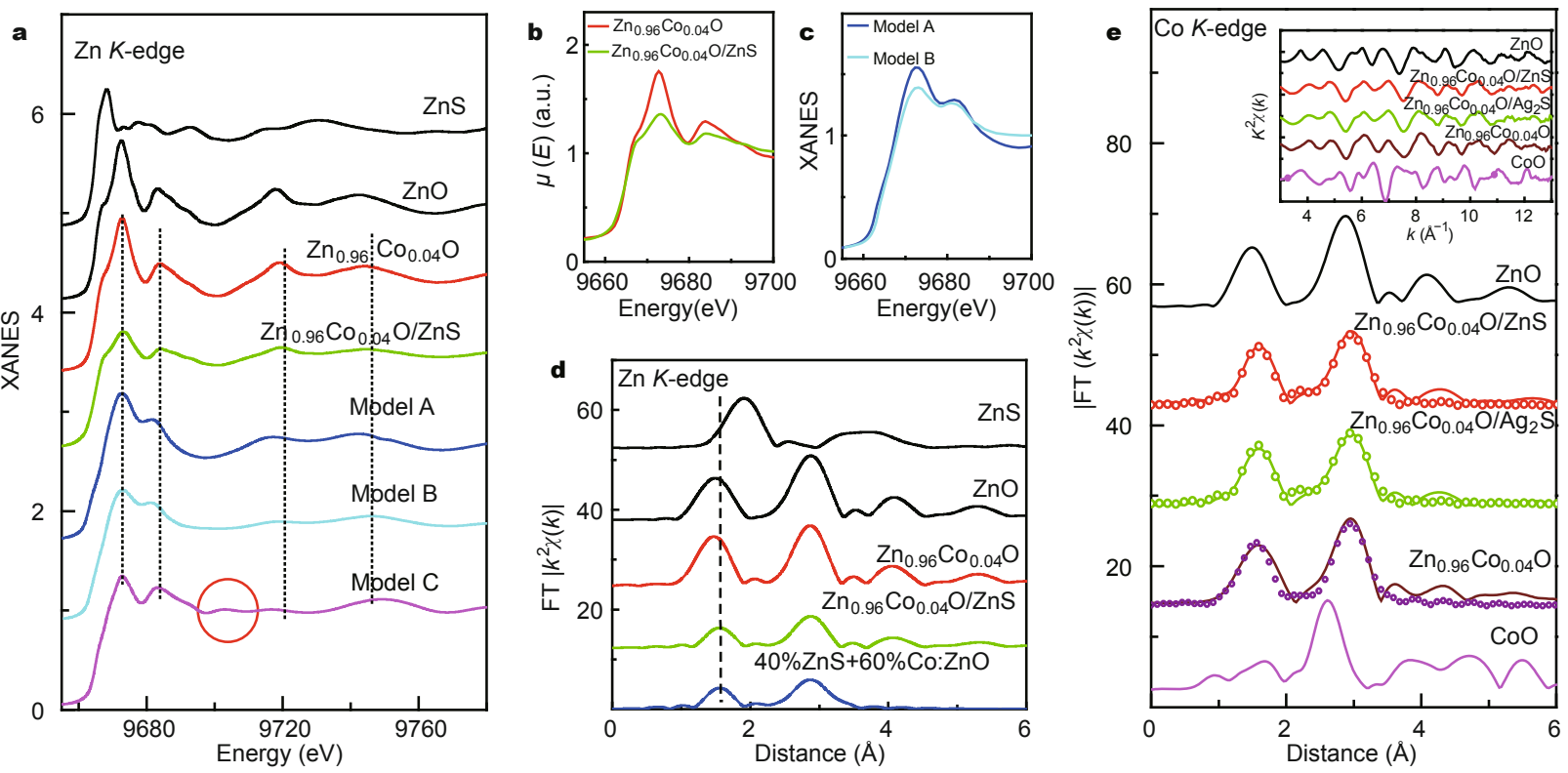

Figure 15 (a) $\mathrm{Zn} K$-edge XANES spectra of $\mathrm{ZnO}, \mathrm{ZnS}, \mathrm{Zn}_{0.96} \mathrm{Co}_{0.04} \mathrm{O}$, and $\mathrm{Zn}_{0.96} \mathrm{Co}_{0.04} \mathrm{O} / \mathrm{ZnS}$, and the calculated spectra for three model structures: Co doped $\mathrm{ZnO}$ (Model A), Co doped ZnO/ZnS (Model B), and ZnO+ZnS (Model C). (b) and (c) are magnified XANES spectra. (d) The FT curves of the $\mathrm{Zn} K$-edge $k^{2} \chi(k)$ functions. (e) The FT curves of the Co $K$-edge $k^{2} \chi(k)$ functions. The empty circles show the fitting results. Reprinted with permission from Ref. [81]. Copyright 2014, American Chemical Society.
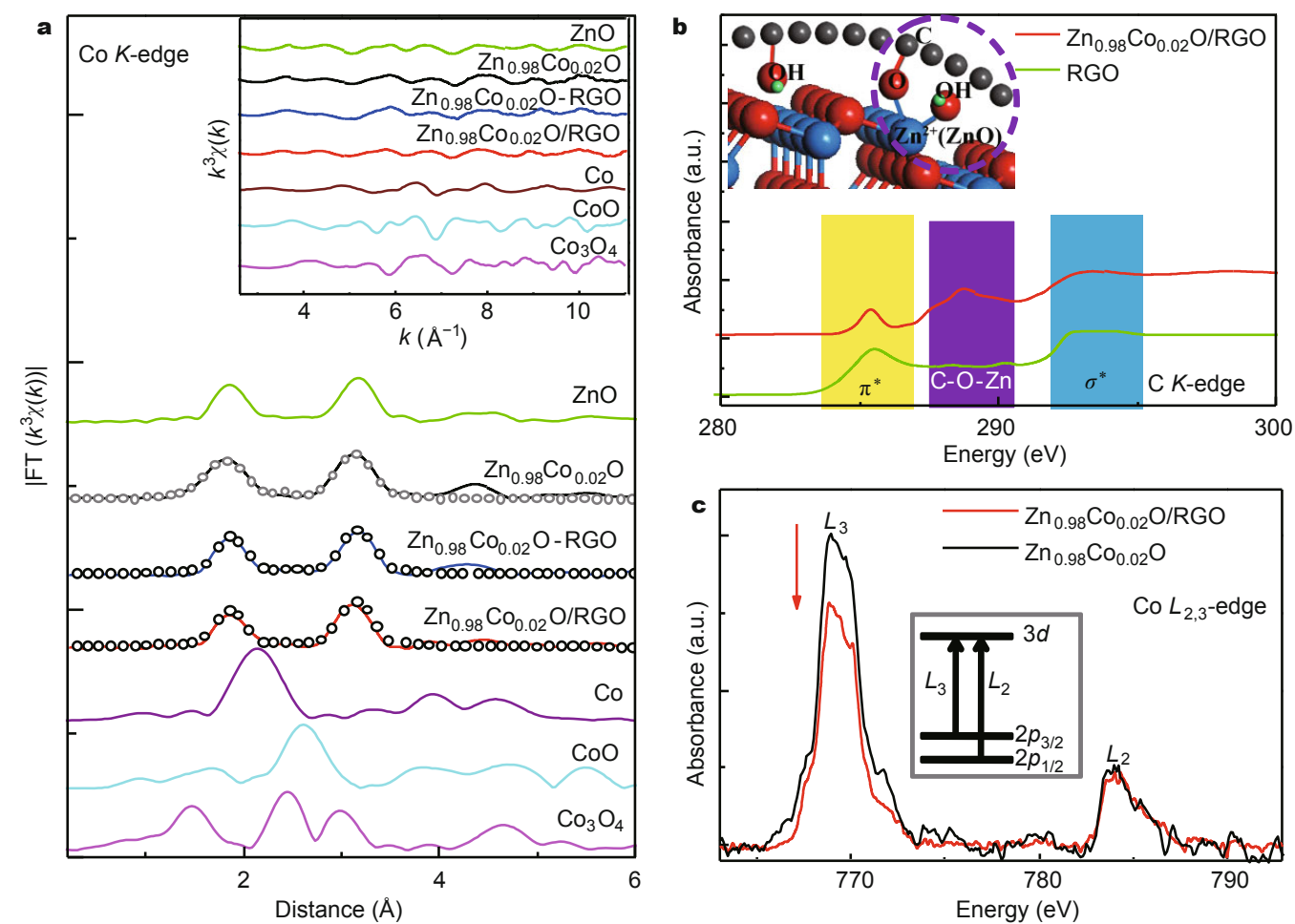

Figure 16 (a) The Co $K$-edge EXAFS-FT curves of the $k^{2} \chi(k)$ functions for $\mathrm{Zn}_{0.98} \mathrm{Co}_{0002} \mathrm{O}-\mathrm{RGO}, \mathrm{Zn}_{0.98} \mathrm{Co}_{002} \mathrm{O} / \mathrm{RGO}, \mathrm{Zn}_{0.98} \mathrm{Co}_{0.02} \mathrm{O}_{4}$ and references. The inset displays the $k^{3} \chi(k)$ functions, and the empty circles show the fitting results. (b) $\mathrm{C} K$-edge XANES spectra. The inset displays the bonds between RGO and Co-doped ZnO. (c) Co $L_{2,3}$-edge XANES spectra. The inset displays the photoelectron transitions of $L_{3}$ and $L_{2}$ at Co $L_{2,3}$-edge. Reprinted with permission from Ref. [82]. Copyright 2014, American Chemical Society. 
show that the characteristic peaks at about 770 and $784 \mathrm{eV}$ arising from Co $2 p \rightarrow 3 d$ electron transitions are decreased in intensity after hybridizing RGO. This implies the higher Co $3 d$ electron occupation in $\mathrm{Zn}_{0.98} \mathrm{Co}_{0.02} \mathrm{O} / \mathrm{RGO}$, primarily due to the charge transfer from $\mathrm{C} 1 s$ to Co $3 d$ states. Such a charge transfer could also be inferred from the Co K-edge XANES spectra. These results suggest strong electronic interactions and electron transfer introduced at the interface of $\mathrm{RGO}$ and $\mathrm{Zn}_{0.98} \mathrm{Co}_{0.02} \mathrm{O}$, intimately related to the observed room temperature ferromagnetic interaction between $\mathrm{Co}^{2+}$ ions in the hybrid structure of Co-doped $\mathrm{ZnO}$ and RGO.

\section{Nanosheets}

Inorganic graphene analogues are a conceptually new class of materials with attractive applications in the next-generation flexible and transparent nanodevices. Our group have conducted a series of studies for various nanosheets materials [83-88], and EXAFS has played important roles in determining their structure and understanding their unique stability.

The first example is the study on the large-area freestanding $\mathrm{ZnSe}$ single layers with four-atomic thickness [83], which were fabricated by virtue of lamellar hybrid
$\left(\mathrm{Zn}_{2} \mathrm{Se}_{2}\right)(\mathrm{pa})$ (pa stands for $n$-propylamine) intermediate. The hybrid ZnSe-pa single layers were initially exfoliated and then pa molecules were removed by heat treatment at $140^{\circ} \mathrm{C}$ for $2 \mathrm{~h}$. To interpret the stability of the $\mathrm{ZnSe}$ single layers, EXAFS data at both $\mathrm{Zn}$ and Se $K$-edges were measured (Figs 17a and b). The Zn K-edge $k \chi(k)$ oscillation curves and their FT curves exhibit different local atomic arrangements of clean $\mathrm{ZnSe}$ single layers with their bulk counterpart and the exfoliated ZnSe-pa single layers, as also confirmed by their Se K-edge data, where the Se-Se peak position is shifted to higher- $R$ side and the peak intensity is greatly reduced. Quantitative results obtained from least-squares curve-fitting suggest that the coordination between $\mathrm{Zn}$ atoms and pa molecules result in slight expansion of the surface $\mathrm{Zn}$-Se bond lengths, but the next nearest $\mathrm{Zn}-\mathrm{Zn}$ distances $(3.85 \AA$ ) are contracted and the Se-Se distances $(4.11 \AA)$ are elongated considerably relative to the bulk value (4.012 $\AA$ ). Such a noticeable distortion on the surface of the exfoliated ZnSe-pa single layers (Fig. 17c) gives rise to a unique electronic structure and an excellent structural stability (Fig. 17d), as revealed by first-principles theoretical calculations. These greatly modified electronic structures then result in an enhanced solar water splitting
Figure 17 (a) $\mathrm{Zn}$ and Se $K$-edge extended XAFS oscillation function $k \chi(k)$ and (b) the corresponding FT curves. (c) Structural model of single-layer $\mathrm{ZnSe}$ sheets, and (d) the calculated Density of States. Reprinted with permission from Ref. [83]. Copyright 2012, Nature Publishing Group.
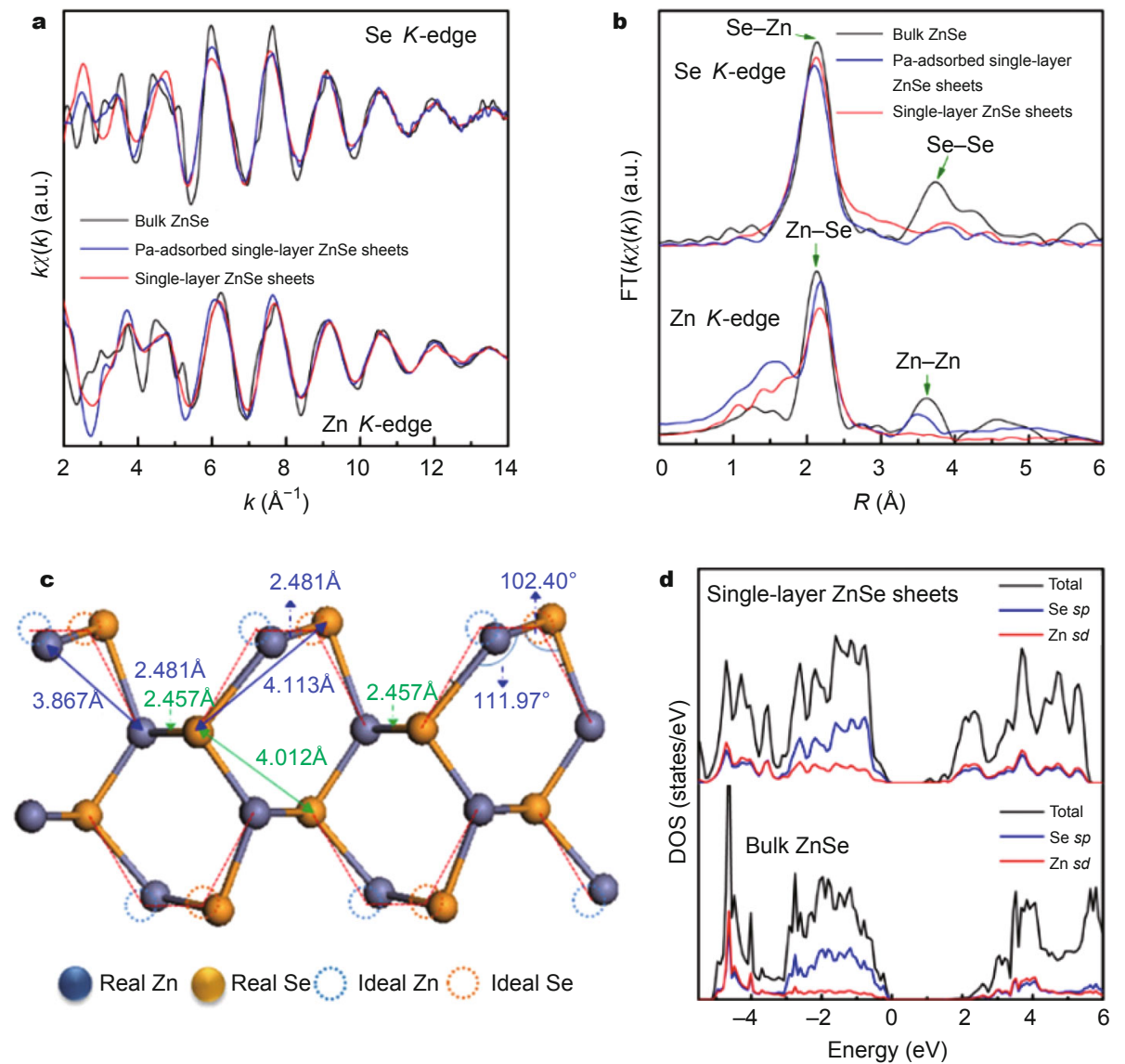
efficiency and photostability of the ZnSe single layers (195 times higher photocurrent density than that of bulk counterpart).

Later, the same authors synthesized freestanding $\mathrm{SnS}_{2}$ nanosheets with three atomic-layer thickness through a convenient and scalable liquid exfoliation strategy [84]. EXAFS and first-principles calculations disclosed that the surface atomic elongation and the structural disorder of the $\mathrm{SnS}_{2}$ single-layers contributed to the excellent structural stability and increased density of states at the valence band edge, enabling the nanosheets to achieve a visible-light water splitting conversion efficiency of $38.7 \%$. Using the scalable interaction/exfoliation strategy, they also fabricated freestanding five-atomic-layer thick $\mathrm{Bi}_{2} \mathrm{Se}_{3}$ nanosheets which possess effective phonon scattering and decreased thermal conductivity, and have an 8-fold higher figure of merit (ZT) relative to the bulk material [85]. EXAFS spectroscopy and first-principles calculations revealed considerable surface distortion of the nanosheets which gives them excellent structural stability and a much increased density of states, resulting in a 2 -fold higher electrical conductivity relative to the bulk material. The same group also studied pits-confined in ultrathin $\mathrm{CeO}_{2}$ nanosheets for studying catalytic centers in carbon monoxide oxidation [86]. EXAFS analysis revealed the average coordination numbers of 4.6 for the artificial three-atom- ic-layer thin $\mathrm{CeO}_{2}$ nanosheets that have approximately $20 \%$ pits occupancy. Density-functional calculations disclose that the four- and five-fold coordinated pit-surrounding cerium sites assume their respective role in carbon monoxide adsorption and oxygen activation, which lowers the activation barrier and avoids catalytic poisoning and leads to reduced conversion temperature and enhanced carbon monoxide catalytic ability.

Recently, based on a 'template-assisted oriented growth' strategy, Cheng and coworkers [87] synthesized half-unitcell nanosheets of a typical transition-metal oxide $\alpha-\mathrm{Fe}_{2} \mathrm{O}_{3}$ that show robust intrinsic ferromagnetism of $0.6 \mu_{\mathrm{B}} /$ atom at $100 \mathrm{~K}$ and remain ferromagnetic at room temperature. The XPS results as shown in Fig. 18a clearly indicate the modified electronic structure of $\mathrm{Fe}$ ions in $\mathrm{a}-\mathrm{Fe}_{2} \mathrm{O}_{3}$ nanosheets than that in $\alpha-\mathrm{Fe}_{2} \mathrm{O}_{3}$ bulk. Next, Fe $K$-edge XANES spectra (Fig. 18b) reveal the difference between the Fe coordination environments in the $\alpha-\mathrm{Fe}_{2} \mathrm{O}_{3}$ nanosheets and bulk. The preedge peak intensity of $\alpha-\mathrm{Fe}_{2} \mathrm{O}_{3}$ nanosheets is $40 \%$ higher than that of bulk $\alpha-\mathrm{Fe}_{2} \mathrm{O}_{3}$, while is similar to that of low-coordinated $\mathrm{Fe}_{3} \mathrm{O}_{4}$ reference compound, suggesting the inherent 5-coordinated Fe ("Fe $\mathrm{F}_{5 \text {-co }}$ ") of nanosheets. However, the white-line peak shape for $\alpha-\mathrm{Fe}_{2} \mathrm{O}_{3}$ nanosheets is close to that of 6-coordinated $\mathrm{Fe}$ (" $\mathrm{Fe}_{6-\mathrm{co}}$ ") in the bulk $\alpha-\mathrm{Fe}_{2} \mathrm{O}_{3}$. Thus, the XANES results reveal that the non-degenerate $\mathrm{Fe}_{5-\text { co }}$ and the $\mathrm{Fe}_{\text {6-co }}$ coordinations probably coexist in the
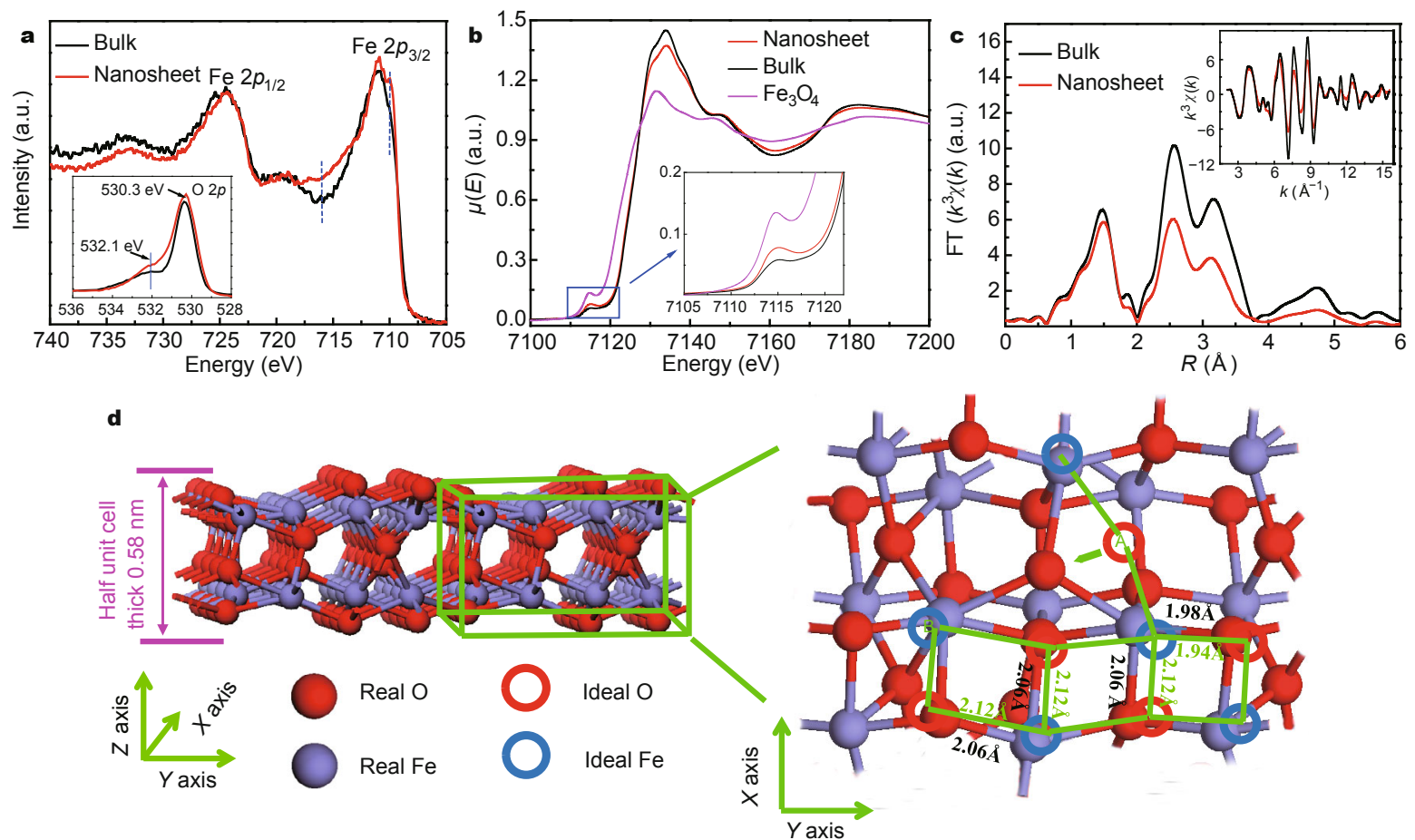

Figure 18 (a) XPS and (b) Fe $K$-edge XANES spectra of $\alpha$ - $\mathrm{Fe}_{2} \mathrm{O}_{3}$ nanosheets, $\alpha-\mathrm{Fe}_{2} \mathrm{O}_{3}$ bulk and $\mathrm{Fe}_{3} \mathrm{O}_{4}$ for reference. (c) FT curves of Fe $K$-edge $k^{3} \chi(k)$ functions of $\alpha-\mathrm{Fe}_{2} \mathrm{O}_{3}$ nanosheets and bulk $\alpha-\mathrm{Fe}_{2} \mathrm{O}_{3}$ (inset: $k^{3} \chi(k)$ curves). (d) Side and top views of the distortion model. Reprinted with permission from Ref. [87]. Copyright 2014, American Chemical Society. 
nanosheets, as is further confirmed by XANES calculations. Furthermore, the Fe $K$-edge EXAFS analysis (Fig. $18 \mathrm{c})$ suggests the retained integrity of hexagonal $\alpha-\mathrm{Fe}_{2} \mathrm{O}_{3}$ structure in the nanosheets, but the $\mathrm{Fe}-\mathrm{Fe}$ second shell is greatly distorted and its structural homogeneity is reduced. The obtained Fe-O coordination number (5.3) is slightly larger than that of the ideal nanosheets, further confirming the $\mathrm{Fe}_{5-\mathrm{co}}+\mathrm{Fe}_{6 \text {-co }}$ mixed coordination. Hence, approximately, $30 \% \mathrm{Fe}$ ions take the 6 -coordinated sites and $70 \%$ of $\mathrm{Fe}$ ions take the 5-coordinated sites. Based on the structural parameters extracted from EXAFS analysis, a structure model for the $\alpha-\mathrm{Fe}_{2} \mathrm{O}_{3}$ nanosheets is established as shown in Fig. 18d. First-principles calculations for this structure model reveal that the efficient breaking of the quantum degeneracy of Fe $3 d$ energy states activates ferromagnetic exchange interaction in these $\mathrm{Fe}_{5-\mathrm{co}}-\mathrm{O}-\mathrm{Fe}_{6-\text { co }}$ ions chains.

\section{In-situ study on the formation processes of nanocrystals and nanoclusters}

Quick XAFS (QXAFS) or energy-dispersive XAFS (ED$\mathrm{XAFS}$ ) techniques have found extensive applications in the study of the nucleation and growth processes of nanoparticles, which is a fundamentally important issue in the controllable synthesis of nanomaterials. Yao and coworkers [89] developed a continuous-flow in-situ QXAFS technique to investigate the nucleation of colloidal $\mathrm{Au}$ nanocrystals reduced by citrate in water in the presence of poly-vinylpyrrolidone (PVP) as surfactant. The reactive solution prepared by the procedure was continuously circulated along the tubes by peristaltic pump during the acquisition of XAFS spectra and flowed into Teflon cell sealed with Kapton windows. Because the static reacted vessel is not suitable for the in-situ measurement directly, recirculation system was used instead to ensure that the samples at the spot were newly formed from the reacted solution to achieve in-situ detection. The recirculation system also has additional advantages that it could avoid the precipitation of particles after a long time reaction and the possible influence of X-ray irradiation in XAFS measurements.

The in situ XAFS spectra clearly show the whole process by reduction of the precursor $\mathrm{AuCl}_{4}^{-}$and the formation of Au nanocrystals (Figs 19a and b). Interestingly, the expansion of the $\mathrm{Au}-\mathrm{Au}$ bond length for the small nuclei at the initial stage implied the formation of novel Au-contained complex which was further confirmed to be the ${ }^{-} \mathrm{Cl}_{3} \mathrm{Au}-\mathrm{AuCl}_{3}{ }^{-}$' dimer cluster by a simulation of the XANES spectra for various cluster configurations in Fig. 19c. The experimental XANES spectra during the first 60 min, for example, the spectrum of $40 \mathrm{~min}$, are more close to that of dimer configuration, and thus can be well fitted (closed squares) by a linear combination of the spectra of the initial state $\left(\mathrm{HAuCl}_{4}\right)$ and intermediate state $\left({ }^{-} \mathrm{Cl}_{3} \mathrm{Au}-\right.$
$\mathrm{AuCl}_{3}{ }^{-}$, dimers) with the partially reduced $\mathrm{AuCl}_{3}{ }^{-}$ions. As the subsequent reduction continued, higher order ' $\mathrm{Au}_{n}$ $\mathrm{Cl}_{n+x}$ ' oligomers were formed in the nucleation stage, and then the growth was accelerated by the coalescence of small clusters. According to the temporal evolutions of the quantitative results, the whole reaction could be classified into three stages: initial nucleation, slow growth, and eventual coalescence (Fig. 19d).

Combining in situ XAFS with other spectroscopy techniques (such as UV-vis) permits the simultaneous determination of the structural information and the species evolution during nanocrystal formation. Weckhuysen and coworkers [90,91] have designed a combined in-situ UVvis/XANES setup to probe the influence of X-rays on aqueous homogeneous copper solutions. They found that that $\mathrm{X}$-rays had effect on the nucleation behavior of nanoparticles due to the radiolysis of water. This effect is mainly localized in a small sample volume within the spot size, with the time scale of several seconds.

Wei and coworkers [92] developed an in-situ measurement combining QXAFS and UV-vis absorption spectroscopies to monitor the nucleation pathways in the solution synthesis of Pt nanocrystals. Figs $20 \mathrm{a}$ and b indicate that the morphologies of the resultant Pt nanocrystals are strongly dependent on the strength of reductants: a wire-like shape for weak reductant of ethylene glycol (EG) and a spherical shape for strong reductant of citric acid (CA). In-situ XAFS and UV-vis probing (Figs 20c-f)) reveal that two different nucleation events occur for the cases of EG and CA reductants, involving the formation of one-dimensional " $\mathrm{Pt}_{n} \mathrm{Cl}_{x}$ " complexes from the polymerization of linear " $\mathrm{Cl}_{3} \mathrm{Pt}-\mathrm{PtCl}_{3}$ "

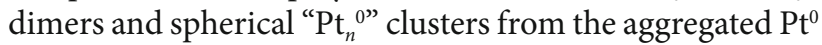
atoms, respectively. The different nucleation pathways then lead to the different morphologies of nanowires and nanospheres. This study suggests that the strength of reductant can be used to tune the nucleation of nanocrystals, and exerts a great influence on the growth dynamics and the final shapes of the nanocrystals.

Using time-resolved QXAFS spectroscopy, Tanaka and coauthors [93-95] also studied the formation mechanism of monolayer-protected gold nanoparticles. Harada and coworkers performed a series of studies on the photoreducted formation processes of different metal nanoparticles. The investigated systems include the formation process of $\mathrm{Au}$ from $\mathrm{AuCl}_{4}^{-}$[96], Pd from $\mathrm{PdCl}_{2}$ [97], Rh from $\mathrm{RhCl}_{3}$ [98], and Pt from $\mathrm{PtCl}_{6}{ }^{2-}[99]$ in the presence of PVP as surfactant.

Fig. 21 show an example of using time-resolved XAFS to monitor the evolutions of photoreduced formation of Pt nanoparticles. Seen from the Pt $L_{3}$-edge XANES spectra in Fig. 21a, the intensity of the white line for precursor $\mathrm{H}_{2} \mathrm{PtCl}_{6}$ solution (before photoirradiation) decreases rapid- 

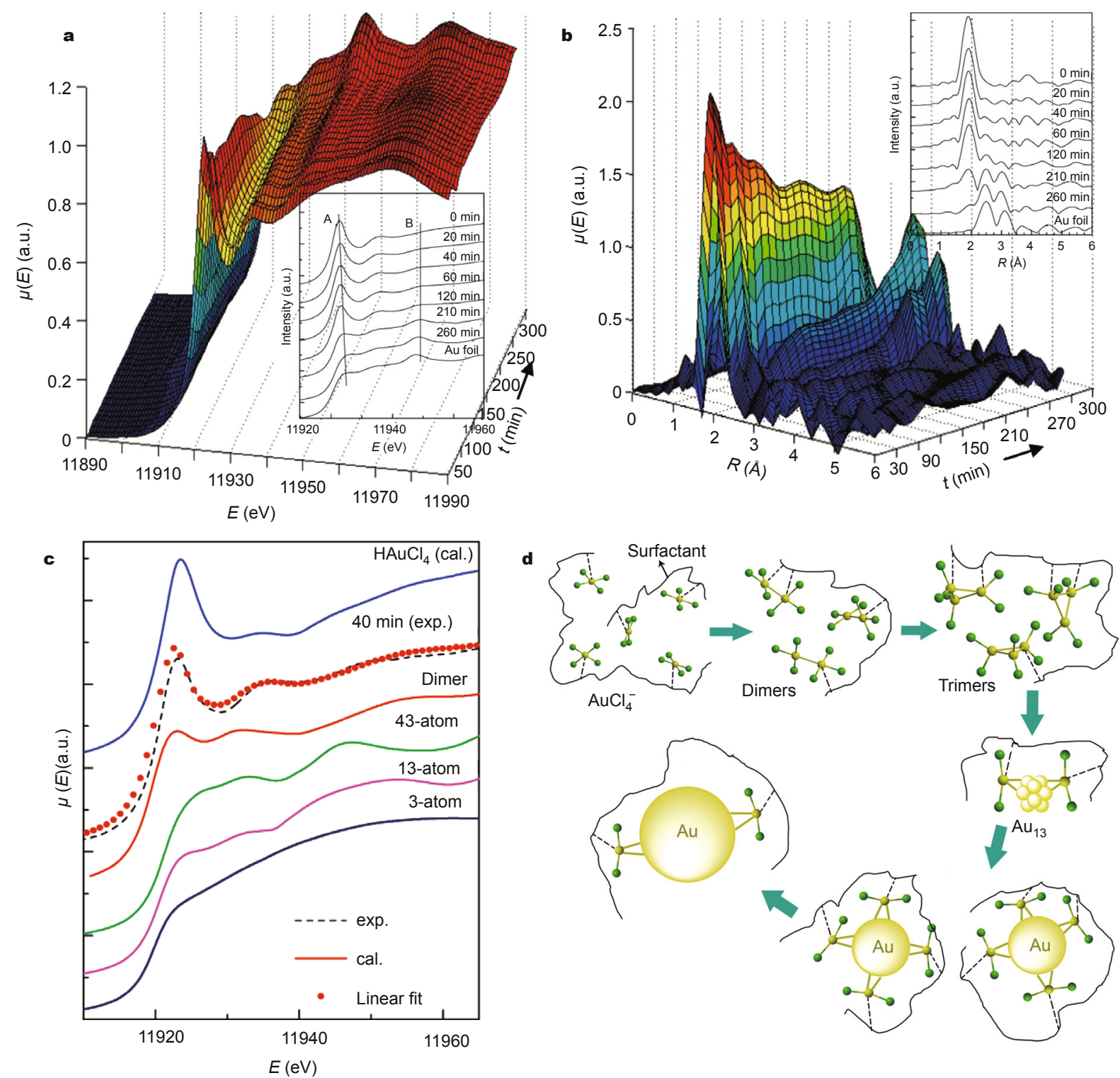

Figure 19 (a) Normalized $\mathrm{Au} L_{3}$-edge in situ XANES, and (b) Fourier transformed $k^{2} \chi(k)$ functions as a function of reaction time. The insets show the typical $\mathrm{Au} L_{3}$-edge XAFS spectra at different reaction time. (c) Comparison of the experimental XANES spectrum at 40 min with calculations for various Au clusters. Reprinted with permission from Ref. [89]. Copyright 2010, American Chemical Society.

ly during the first 10 min and then gradually decreases in course of reduction time. The isosbestic point shifts from 11,569 to $11,568 \mathrm{eV}$ accordingly, especially in the reduction time between 10 and $40 \mathrm{~min}$, indicating the stepwise transformation of one species to another $\left(\mathrm{Pt}^{4+} \rightarrow \mathrm{Pt}^{2+} \rightarrow \mathrm{Pt}^{0}\right)$. Meanwhile, the maximum peak position $(11,590 \mathrm{eV})$ attributed to the metallic Pt-Pt bond increases with the increase of reduction time, suggesting the accumulation of small $\mathrm{Pt}$ nanoparticles. When the photoirradiation time increases, the $\mathrm{Pt}-\mathrm{Cl}$ peak located at $1.96 \AA$ in the EXAFS-FT curves (Fig. 21b) gradually decreases in intensity, and the other peak at around $2.57 \AA$ assigned to a metallic Pt-Pt bond becomes more and more intensive. Based on these spectral observations as well as TEM and UV-vis absorption spectra, the authors proposed a general process involving the initial formation of $\mathrm{Pt}$ nuclei (i.e., $\left(\mathrm{Pt}^{0}\right)_{m}$ nucleates approximately $m=4$ ), followed by the aggregative particle growth with the autocatalytic reduction of $\mathrm{Pt}$ ionic species on the surface of Pt nuclei to produce Pt nanoparticles (Fig. 21c). Comparison of the different photoreduction reactions for various nanaoparticles indicates that the reductions of the precursor metal species with high oxidation state possessed an induction period before the onset of metal particle formation. In these photoreduction reactions, the onset of 
a
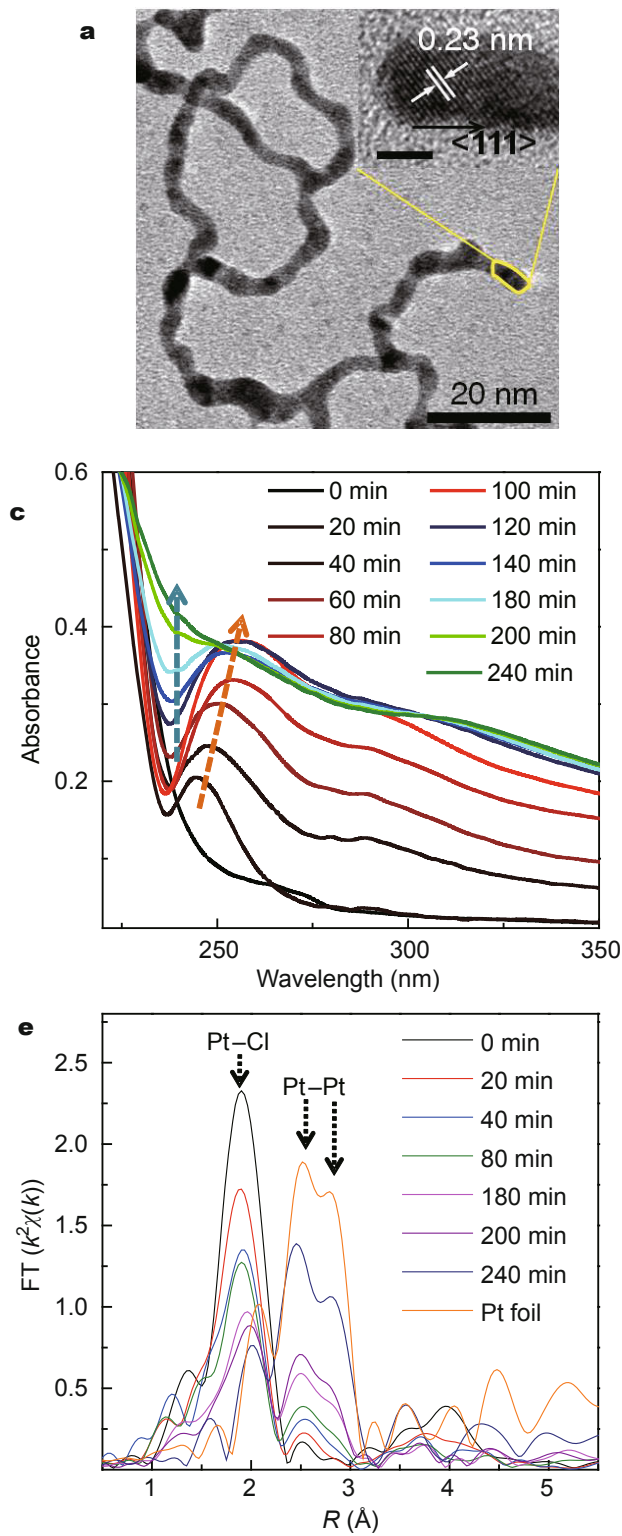

b
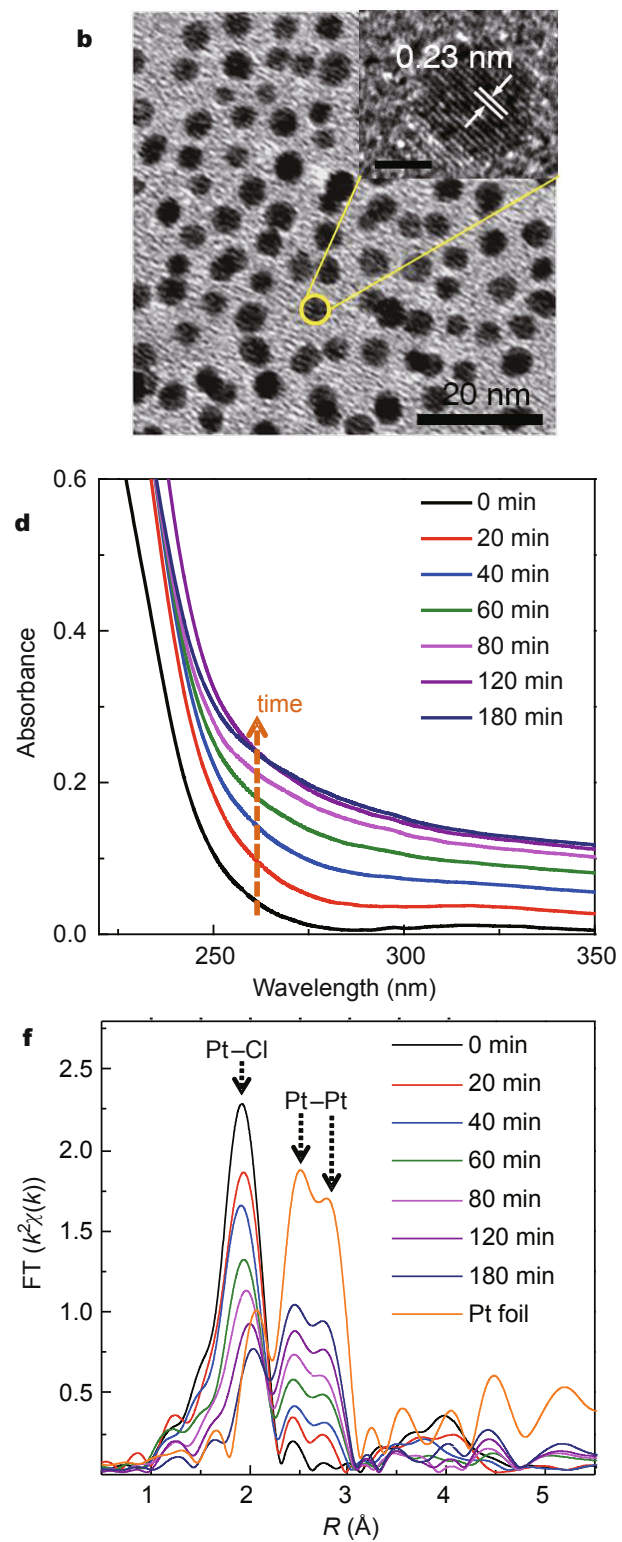

Figure 20 Two representative mophologies, i.e., (a) nanowires and (b) nanospheres synthesized by using ethylene glycol and citric acid, respectively. Temporal evolution of UV-Vis absorption spectra of Pt colliod solutions for the $\mathrm{K}_{2} \mathrm{PtCl}_{4}$ reduced by (c) ethylene glycol and (d) citric acid. (e and f) Time variations in the Fourier transformed $k^{2} \chi(k)$ spectra during the reaction processes of the $\mathrm{K}_{2} \mathrm{PtCl}_{4}$ reduced by ethylene glycol and citric acid, respectively. Reprinted with permission from Ref. [92]. Copyright 2012, American Chemical Society.

metal-metal bond signal occurs after the fully reduction of the metal ionic species into zero-valent atoms.

\section{Nanostructured catalysts under operando conditions}

It is widely recognized that the catalysis cannot be understood without spectroscopic techniques [100]. In-situ spectroscopic characterization of catalysts under working (operando) conditions could bridge the gap between structure-performance relationships, due to the simultaneous measurement of the catalytic activity/selectivity in a kinetically relevant in-situ reactor cell. Iwasawa and coworkers [101] developed a novel time-gating quick XAFS (TG-QXAFS) technique with $1 \mathrm{~s}$ time resolution and an energy-dispersive XAFS system with $4 \mathrm{~ms}$ time resolution. Using these techniques, they first conducted a study on the time-resolved dynamic surface events on the $\mathrm{Pt} / \mathrm{C}$ cathode in a fuel cell under operando conditions. Figs $22 \mathrm{a}$ and $\mathrm{b}$ show a series of TG-QEXAFS $k^{3} \chi(k)$ oscillations at Pt $L_{3}$ edge for a $\mathrm{Pt} / \mathrm{C}$ cathode catalyst during a voltage-stepped process from 1.0 to $0.4 \mathrm{~V}$ and the FT curves from 0.4 to 

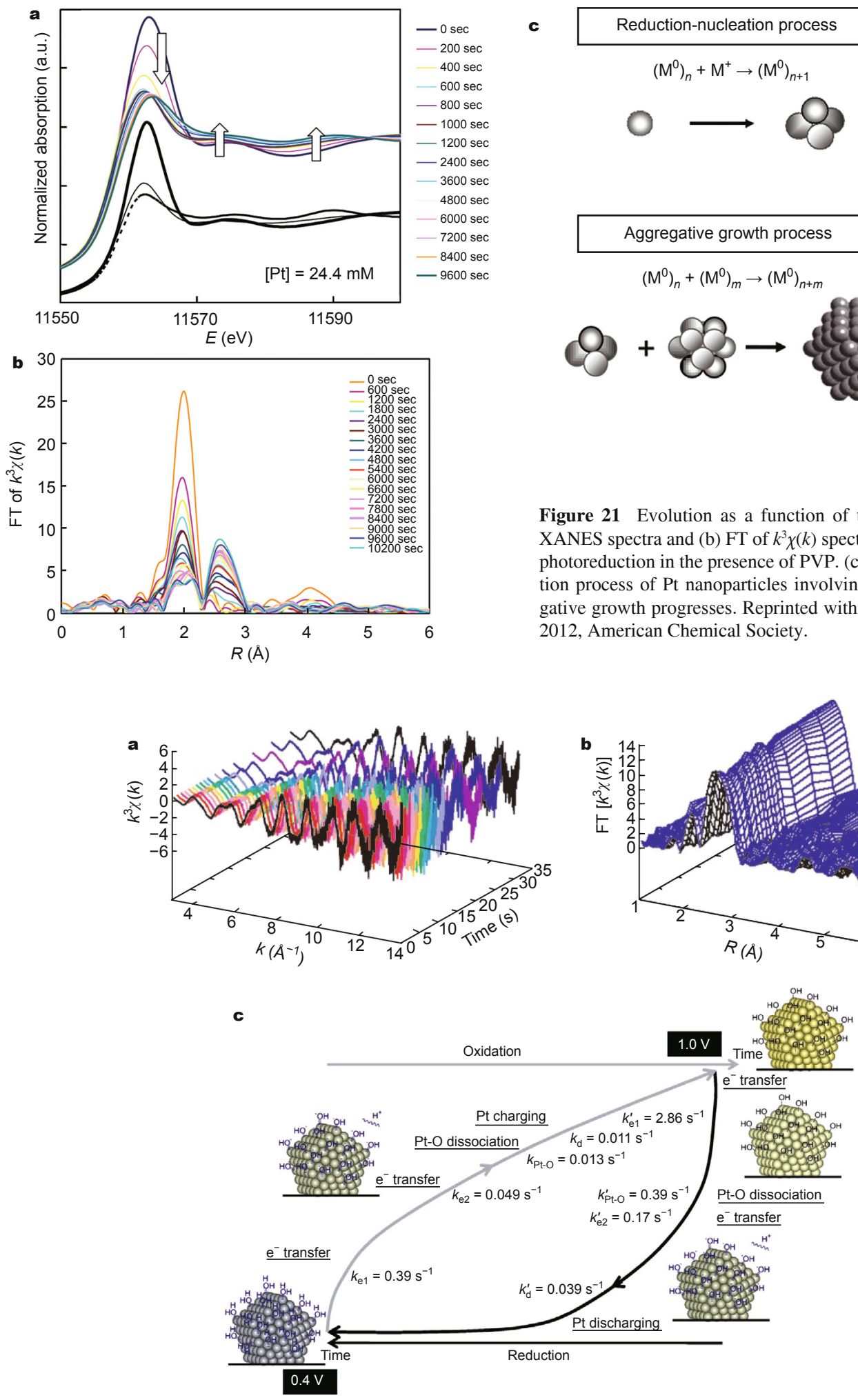

Figure 22 (a) A series of TG-QEXAFS oscillations of a reduction process $(1.0 \rightarrow 0.4 \mathrm{~V})$, and (b) the Fourier transforms of an oxidation process $(0.4 \rightarrow 1.0$ $\mathrm{V}$ ) at $\mathrm{Pt} L_{3}$-edge in a Pt/C fuel cell measured at $333 \mathrm{~K}$. (c) Reaction mechanism for changes in the Pt/C fuel-cell catalysts in cell voltages of $0.4 / 1.0 \mathrm{~V}$ under $\mathrm{H}_{2}$ (anode) and $\mathrm{N}_{2}$ (cathode). Reprinted with permission from Ref. [101]. Copyright 2007, Wiley-Blackwell. 
1.0 V. All the TG-QEXAFS spectra have good enough data quality for curve-fitting analysis to evaluate the numbers and the lengths of chemical bonds on the Pt cathode catalyst. Based on the obtained parameters (electrochemical charges, numbers and lengths of $\mathrm{Pt}-\mathrm{Pt}$ and $\mathrm{Pt}-\mathrm{O}$ bonds, electron density of $\mathrm{Pt} d$ orbitals), they determined eight rate constants for the oxidation and reduction processes (Fig. 22c). These eight rate constants, significantly different from each other, are related to the electron transfer, structural change, and $d$-electron density change at the $\mathrm{Pt} / \mathrm{C}$ cathode catalyst. Besides, a hysteresis loop for the structural changes in the Pt cathode particles in the oxidation and reduction processes is observed.

Iwasawa's group [102] also studied the reductive formation and oxidative phase separation of $\gamma-\mathrm{Al}_{2} \mathrm{O}_{3}$ supported $\mathrm{Pt}_{3} \mathrm{Sn}$ nanoparticles during the reduction and oxidation processes at $673 \mathrm{~K}$. Fig. 23a shows a series of time-resolved XANES spectra at $\mathrm{Pt} L_{3}$-edge, which were measured every $0.1 \mathrm{~s}$, in the reduction process of the oxidized $\mathrm{Pt}_{3} \mathrm{Sn} / \gamma$ $\mathrm{Al}_{2} \mathrm{O}_{3}$ catalyst at $673 \mathrm{~K}$ under a $\mathrm{H}_{2}$ pressure of $23.6 \mathrm{kPa}$. The white line intensity decreased after $\mathrm{H}_{2}$ admission to the in-situ XAFS cell, and its decay against the reduction time was plotted in Fig. 23b. The change in the white line intensity was nearly completed in $2 \mathrm{~s}$. Because the variation of the white line with reduction time is equivalent to the variation of the amount of $\mathrm{PtO}$ in the catalyst, the dynamic behavior and the structural kinetics of the PtO nanoparticles in the reduction process could be determined. Fig.
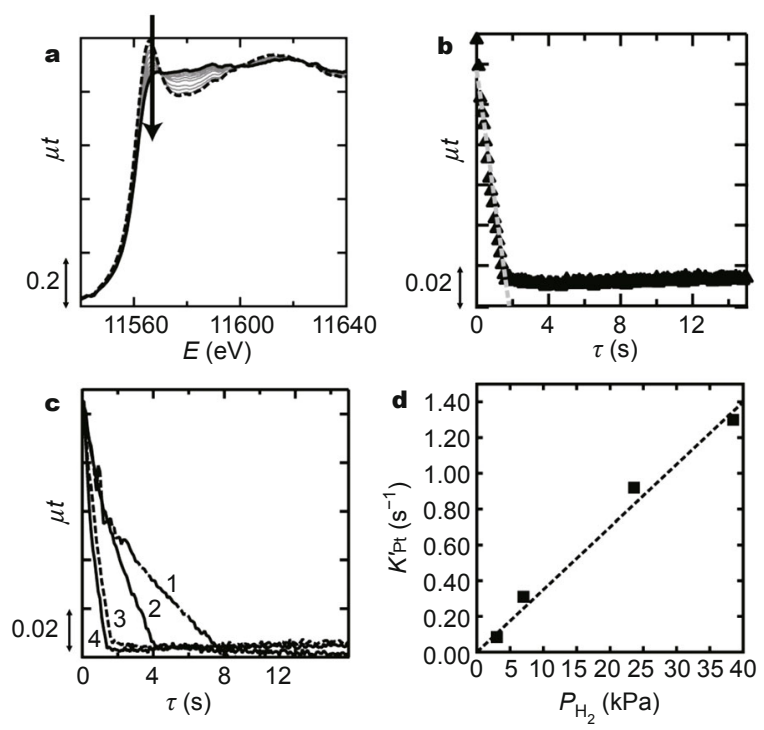

Figure 23 (a) In situ time-resolved $\mathrm{Pt} L_{3}$-edge XANES spectra in the reduction process of the oxidized $\mathrm{Pt}_{3} \mathrm{Sn} / \gamma-\mathrm{Al}_{2} \mathrm{O}_{3}$ catalyst at $673 \mathrm{~K}$ under $P_{\mathrm{H} 2}=23.6 \mathrm{kPa}$. (b) Time profile of the change in the white line intensity. (c) Change of the white line intensity over time at different $\mathrm{H}_{2}$ pressures. (d) Dependence on $P_{\mathrm{H} 2}$ of the rate constants in the reduction at $673 \mathrm{~K}$. Reprinted with permission from Ref. [102]. Copyright 2011, American Chemical Society.
$23 c$ displays the change in the white line intensity in the reduction of the catalyst at different pressures of $\mathrm{H}_{2}$, and the obtained rate constants are plotted in Fig. 23d against the $\mathrm{H}_{2}$ pressures. At a very low $\mathrm{H}_{2}$ pressure like $3.0 \mathrm{kPa}$, the white line intensity varied with time in a two-step process, where the first reduction step was followed by a slower second step. Further in-situ time-resolved XAFS measurements at $\mathrm{Pt} L_{3}$-edge and $\mathrm{Sn} K$-edge reveal that the oxidation of $\mathrm{Pt}_{3} \mathrm{Sn}$ nanoparticles on $\gamma-\mathrm{Al}_{2} \mathrm{O}_{3}$ with $\mathrm{O}_{2}$ at $673 \mathrm{~K}$ proceed by three successive steps via two intermediates to form $\mathrm{PtO}$ core nanoparticles with $\mathrm{SnO}_{2}$ shells, whereas the reduction of the oxidized nanoparticles with $\mathrm{H}_{2}$ at $673 \mathrm{~K}$ proceed as a single process with similar rate constants at Pt and $\mathrm{Sn}$ sites.

Using the established time-resolved XAFS method, the Iwasawa's group [103] also studied the dynamic structural changes and kinetics of a $\operatorname{Re}_{10}$ cluster catalyst in the direct phenol synthesis from benzene and $\mathrm{O}_{2}$. The real-time XAFS measurements monitoring the structural transformation of active $\mathrm{Re}_{10}$ clusters to inactive Re monomers suggested the direct transformation of $\mathrm{Re}_{10}$ cluster to the Re monomers, showing a first order reaction to the $\mathrm{Re}_{10}$ cluster quantity. Besides, they used the time-resolved XAFS with a $0.5 \mathrm{~s}$ time resolution to study the structural kinetics of surface events on $\mathrm{Pt}_{3} \mathrm{Co} / \mathrm{C}$ cathode catalyst in a polymer electrolyte fuel cell under operando conditions [104].

Besides the Iwasawa's group, Frenkel and cooperators have performed a series of studies on the application of XAFS (and other complementary techniques) to heterogeneous catalysis, like $\mathrm{CuO} / \mathrm{CeO}_{2}$ [105], $\mathrm{Ce}_{1-x} \mathrm{Cu}_{x} \mathrm{O}_{2}$ [106], $\mathrm{CeO}_{2-x} / \mathrm{CuO}$ [58], and $\mathrm{AuO}_{x} / \mathrm{CeO}_{2}$ [107] WGS catalysts. Their studies aim to obtain a systematic understanding of the structural, electronic, and chemical properties of these catalysts under the operando conditions, in order to reveal the nature of the active phase in metal/oxide WGS catalysts and the WGS reaction mechanism. Some of these studies have been reviewed in subsection of "Oxide nanoparticles" in this paper, and interested readers are referred to a few review articles $[108,109]$ on the capabilities, challenges, and opportunities of the in-situ/operando catalytic studies.

\section{OUTLOOK AND PERSPECTIVE}

XAFS has been developed into a mature and powerful technique for studies on the local atomic/electronic structures of a specific type of atoms in condensed matters. The recent significant advances in theory and experimental methods have made it more and more widely and extensively used. As many new generations of synchrotron radiation light sources and more advanced XAFS beamlines are available, undoubtedly the range of XAFS applications will continue extending. Especially, in the study of nanomaterials, with the improvement of the time-resolved XAFS technique, the in-situ probing of the formation processes of nanomaterials 
and their behaviors under operando conditions will play valuable roles in prompting the prosperity of the nanoscience and nanotechnology. It is worthy to note that the local structure from XAFS analysis is always about the average structure. Only a synergetic utilization of different methods bringing complementary information together enables a comprehensive and detailed understanding of the materials under investigation. Along this way, combining two or more in-situ characterization methods in one setup has been greatly advanced and will still continue to be developed.

\section{Received 27 February 2015; accepted 17 March 2015;} published online 10 April 2015

1 Drexler KE. Engines of Creation: The Coming Era of Nanotechnology. New York: Doubleday, 1986

2 Nanoscience and nanotechnologies: opportunities and uncertainties. London: The Royal Society and The Royal Academy of Engineering, 2004

3 Binnig G, Quate CF, Gerber C. Atomic force microscope. Phys Rev Lett, 1986, 56: 930-933

4 Binnig G, Rohrer H. Scanning tunneling microscopy. IBM J Res Dev, 1986, 30: 355-369

5 Koningsberger DC, Prins R. X-Ray Absorption: Principles, Applications, Techniques of EXAFS, SEXAFS and XANES. New York: Wiley, 1988

6 Bunker G. A Practical Guide to X-ray Absorption Fine Structure Spectroscopy. Cambridge: Cambridge University Press, 2010

7 Dent AJ. Development of time-resolved XAFS instrumentation for quick EXAFS and energy-dispersive EXAFS measurements on catalyst systems. Top Catal, 2002, 18: 27-35

8 Pasquarello A, Petri I, Salmon PS, et al. First solvation shell of the $\mathrm{Cu}(\mathrm{II})$ aqua ion: evidence for fivefold coordination. Science, 2001, 291: 856-859

9 Zhang XW, Yan XJ, Zhou ZR, et al. Arsenic trioxide controls the fate of the PML-RAR alpha oncoprotein by directly binding PML. Science, 2010, 328: 240-243

10 Chen LX, Jager WJH, Jennings G, et al. Capturing a photoexcited molecular structure through time-domain X-ray absorption fine structure. Science, 2001, 292: 262-264

11 Bianconi A, Saini NL, Lanzara A, et al. Determination of the local lattice distortions in the $\mathrm{CuO}_{2}$ plane of $\mathrm{La}_{1.85} \mathrm{Sr}_{0.15} \mathrm{CuO}_{4}$. Phys Rev Lett, 1996, 76: 3412-3415

12 Fricke $\mathrm{H}$. The K-characteristic absorption frequencies for the chemical elements magnesium to chromium. Phys Rev, 1920, 16 : 202-215

13 Hertz GZ. Absorption limits of the $L$-series. Zeitschrift Fur Physik, 1920, 3: 19-25

14 Kronig RD. On the theory of fine structure in the X-ray absorption spectra. Zeitschrift Fur Physik, 1931, 70: 317-323

15 Kronig RD. On the theory of fine structure in the X-ray absorption spectrum 3. Zeitschrift Fur Physik, 1932, 75: 468-475

16 Kronig RD. On the theory of fine structure in the X-ray absorption spectrum. 2. Zeitschrift Fur Physik, 1932, 75: 191-210

17 Sayers DE, Stern EA, Lytle FW. New technique for investigating noncrystalline structures: Fourier analysis of extended X-rayabsorption fine structure. Phys Rev Lett, 1971, 27: 1204-1207

18 Rehr JJ, Stern EA, Martin RL, Davidson ER. Extended X-rayabsorption fine-structure amplitudes-Wave-function relaxation and chemical effects. Phys Rev B, 1978, 17: 560-565

19 Bunker G. Application of the ratio method of exafs analysis to dis- ordered-systems. Nucl Instrum Meth A, 1983, 207: 437-444

20 Tranquada JM, Ingalls R. Extended X-ray-absorption fine-structure study of anharmonicity in CuBr. Phys Rev B, 1983, 28: 3520-3528

21 Crozier ED, Seary AJ. Asymmetric effects in the extended X-ray absorption fine-structure analysis of solid and liquid zinc. Can J Phys, 1980, 58: 1388-1399

22 Wei SQ, Oyanagi H, Liu WH, et al. Local structure of liquid gallium studied by X-ray absorption fine structure. J Non-Cryst Solids, 2000, 275: 160-168

23 Lee PA, Pendry JB. Theory of extended X-ray absorption fine-structure. Phys Rev B, 1975, 11: 2795-2811

24 Rehr JJ, Albers RC. Scattering-matrix formulation of curvedwave multiple-scattering theory: application to X-ray-absorption fine-structure. Phys Rev B, 1990, 41: 8139-8149

25 Rehr JJ, Deleon JM, Zabinsky SI, Albers RC. Theoretical X-ray absorption fine-structure standards. J Am Chem Soc, 1991, 113: 5135-5140

26 Rehr JJ, Albers RC. Theoretical approaches to X-ray absorption fine structure. Rev Mod Phys, 2000, 72: 621-654

27 Fujikawa T, Yiwata N. A new approach to full multiple-scattering XAFS calculation. Surf Sci, 1996, 357: 60-64

28 Ankudinov AL, Ravel B, Rehr JJ, Conradson SD. Real-space multiple-scattering calculation and interpretation of X-ray-absorption near-edge structure. Phys Rev B, 1998, 58: 7565-7576

29 Natoli CR, Misemer DK, Doniach S, Kutzler FW. First-principles calculation of X-ray absorption-edge structure in molecular clusters. Phys Rev A, 1980, 22: 1104-1108

30 Binsted N. EXCURV98: CCLRC Daresbury Laboratory Computer Program. 1998; Available from: http://srs.dl.ac.uk/XRS/Computing/Programs/excurv97/intro.html

31 Filipponi A, Di Cicco A, Natoli CR. X-ray-absorption spectroscopy and $\mathrm{n}$-body distribution functions in condensed matter. I. Theory. Phys Rev B, 1995, 52: 15122-15134

32 Blaha P, Schwarz K, Madsen GKH, Kvasnicka D, Luitz J. WIEN2k: An Augmented Plane Wave plus Local Orbitals Program for Calculating Crystal Properties. Karlheinz Schwarz, Technische Universität Wien, 2001

33 Zhong WJ, He B, Li Z, Wei SQ. USTCXAFS 2.0 software packages (in Chinese). J Univ Sci Technol China, 2001, 31: 228-233

34 Baberschke K. Recent progress in low-Z SEXAFS. Phys B, 1989, 158: 19-24

35 Magnan H, Chandesris D, Rossi G, et al. Determination of the local order in amorphous cobalt films. Phys Rev B, 1989, 40: 9989-9992

36 Heckmann O, Magnan H, Lefevre P, Chandesris D, Rehr JJ. Crystallographic structure of cobalt filsm on $\mathrm{Cu}(001)$ : elastic-deformation to a tetragonal structure. Surf Sci, 1994, 312: 62-72

37 Diaz-Moreno S. XAFS data collection: an integrated approach to delivering good data. J Synchrotron Rad, 2012, 19: 863-868

38 Stern EA, Kim K. Thickness effect on the extended-X-ray-absorption-fine-structure amplitude. Phys Rev B, 1981, 23: 3781-3787

39 Henke BL, Gullikson EM, Davis JC. X-ray interactions: photoabsorption, scattering, transmission, and reflection at $E=50-30,000$ eV, $Z=1-92$. Atom Data Nucl Data Tables, 1993, 54: 181-342

40 Chantler CT. Theoretical form-factor, attenuation and scattering tabulation for $Z=1-92$ from $E=1-10 \mathrm{eV}$ to $E=0.4-1.0 \mathrm{meV}$. J Phys Chem Ref Data, 1995, 24: 71-591

41 Troger L, Arvanitis D, Baberschke K, et al. Full correction of the self-absorption in soft-fluorescence extended X-ray-absorption fine-structure. Phys Rev B, 1992, 46: 3283-3289

42 Booth $\mathrm{CH}$, Bridges $\mathrm{F}$. Improved self-absorption correction for fluorescence measurements of extended X-ray absorption fine-structure. Phys Scr, 2005, T115: 202-204

43 Lytle FW, Greegor RB, Sandstrom DR, et al. Measurement of soft$\mathrm{X}$-ray absorption-spectra with a fluorescent ion-chamber detector. Nucl Instrum Meth A, 1984, 226: 542-548 
44 Oyanagi H, Fonne C, Gutknecht D, et al. Ge pixel array detector for high throughput X-ray spectroscopy. Nucl Instrum Meth A, 2003, 513: 340-344

45 Oyanagi H, Sakamoto K, Shioda R, Kuwahara Y, Haga K. Ge overlayers on $\mathrm{Si}(001)$ studied by surface-extended X-ray-absorption fine structure. Phys Rev B, 1995, 52: 5824-5829

46 Yin Y, Alivisatos AP. Colloidal nanocrystal synthesis and the organic-inorganic interface. Nature, 2005, 437: 664-670

47 Donega CD. Synthesis and properties of colloidal heteronanocrystals. Chem Soc Rev, 2011, 40: 1512-1546

48 Vericat C, Vela ME, Benitez G, Carro P, Salvarezza RC. Self-assembled monolayers of thiols and dithiols on gold: new challenges for a well-known system. Chem Soc Rev, 2010, 39: 1805-1834

49 Xia YN, Cobley CM, Chen JY, Cho EC, Wang LV. Gold nanostructures: a class of multifunctional materials for biomedical applications. Chem Soc Rev, 2011, 40: 44-56

50 Zhang P, Sham TK. X-ray studies of the structure and electronic behavior of alkanethiolate-capped gold nanoparticles: the interplay of size and surface effects. Phys Rev Lett, 2003, 90: 245502

51 Sanchez SI, Menard LD, Bram A, et al. The emergence of nonbulk properties in supported metal clusters: negative thermal expansion and atomic disorder in Pt nanoclusters supported on gamma- $\mathrm{Al}_{2} \mathrm{O}_{3}$. J Am Chem Soc, 2009, 131: 7040-7054

52 Small MW, Sanchez SI, Marinkovic NS, Frenkel AI, Nuzzo RG. Influence of adsorbates on the electronic structure, bond strain, and thermal properties of an alumina-supported Pt catalyst. ACS Nano, 2012, 6: 5583-5595

53 Jin RC. Quantum sized, thiolate-protected gold nanoclusters. Nanoscale, 2010, 2: 343-362

54 Li G, Jin RC. Atomically precise gold nanoclusters as new model catalysts. Acc Chem Res, 2013, 46: 1749-1758

55 Lu YZ, Chen W. Sub-nanometre sized metal clusters: from synthetic challenges to the unique property discoveries. Chem Soc Rev, 2012, 41: 3594-3623

56 Spivey JJ, Krishna KS, Kumar C, et al. Synthesis, characterization, and computation of catalysts at the center for atomic-level catalyst design. J Phys Chem C, 2014, 118: 20043-20069

57 Liu J, Krishna KS, Losovyj YB, et al. Ligand-stabilized and atomically precise gold nanocluster catalysis: a case study for correlating fundamental electronic properties with catalysis. Chem-Euro J, 2013, 19: 10201-10208

58 Barrio L, Estrella M, Zhou G, et al. Unraveling the active site in copper-ceria systems for the water-gas shift reaction: in situ characterization of an inverse powder $\mathrm{CeO}_{2-x} / \mathrm{CuO}-\mathrm{Cu}$ catalyst. J Phys Chem C, 2010, 114: 3580-3587

59 Patlolla A, Carino EV, Ehrlich SN, Stavitski E, Frenkel AI. Application of operando XAS, XRD, and Raman spectroscopy for phase speciation in water gas shift reaction catalysts. ACS Catal, 2012, 2: 2216-2223

60 Oyanagi H, Sakamoto K, Shioda R, Sakamoto T. Ge epitaxial overlayers on $\mathrm{Si}(001)$ studied by surface-sensitive X-ray-absorption fine-structure: evidence for strain-induced surface rearrangement. Jpn J Appl Phys, 1994, 33: 3545-3552

61 Oyanagi H, Sakamoto K, Shioda R, Kuwahara Y, Haga K. Ge overlayers on $\mathrm{Si}(001)$ studied by surface-extended X-ray-absorption fine-structure. Phys Rev B, 1995, 52: 5824-5829

62 Pearsall TP, Bevk J, Feldman LC, et al. Structurally induced optical-transitions in Ge-Si superlattices. Phys Rev Lett, 1987, 58: 729-732

63 Wei SQ, Oyanagi H, Sakamoto K, Takeda Y, Pearsall TP. Local structure of $\left(\mathrm{Ge}_{4} \mathrm{Si}_{4}\right)_{5}$ monolayer strained-layer superlattice probed by fluorescence X-ray absorption fine structure. Phys Rev B, 2000, 62: $1883-1888$

64 Kamenev BV, Tsybeskov L, Baribeau JM, Lockwood DJ. Photoluminescence and Raman scattering in three-dimensional $\mathrm{Si} / \mathrm{Si}_{1-x} \mathrm{Ge}_{x}$ nanostructures. Appl Phys Lett, 2004, 84: 1293-1295

65 Sun ZH, Wei SQ, Kolobov AV, Oyanagi H, Brunner K. Short-range order structures of self-assembled Ge quantum dots probed by multiple-scattering extended X-ray absorption fine structure. Phys Rev B, 2005, 71: 245334

66 Wolf SA, Awschalom DD, Buhrman RA, et al. Spintronics: a spinbased electronics vision for the future. Science, 2001, 294: 14881495

67 Žutić I, Fabian J, Das Sarma S. Spintronics: fundamentals and applications. Rev Mod Phys, 2004, 76: 323-410

68 Ohno H. Making nonmagnetic semiconductors ferromagnetic. Science, 1998, 281: 951-956

69 Dietl T, Ohno H, Matsukura F, Cibert J, Ferrand D. Zener model description of ferromagnetism in zinc-blende magnetic semiconductors. Science, 2000, 287: 1019-1022

70 Liu C, Yun F, Morkoc H. Ferromagnetism of $\mathrm{ZnO}$ and GaN: a review. J Mater Sci-Mater El, 2005, 16: 555-597

71 Wei SQ, Yan WS, Sun ZH, et al. Direct determination of Mn occupations in $\mathrm{Ga}_{1-x} \mathrm{Mn}_{x} \mathrm{~N}$ dilute magnetic semiconductors by X-ray absorption near-edge structure spectroscopy. Appl Phys Lett, 2006, 89: 121901

72 He B, Zhang XY, Wei SQ, et al. Local structure around Mn atoms in cubic $(\mathrm{Ga}, \mathrm{Mn}) \mathrm{N}$ thin films probed by fluorescence extended X-ray absorption fine structure. Appl Phys Lett, 2006, 88: 051905

73 Kunisu M, Oba F, Ikeno H, Tanaka I, Yamamoto T. Local environment of $\mathrm{Mn}$ dopant in $\mathrm{ZnO}$ by near-edge $\mathrm{X}$-ray absorption fine structure analysis. Appl Phys Lett, 2005, 86: 121902

74 Sun ZH, Yan WS, Zhang GB, et al. Evidence of substitutional Co ion clusters in $\mathrm{Zn}_{1-x} \mathrm{Co}_{x} \mathrm{O}$ dilute magnetic semiconductors. Phys Rev B, 2008, 77: 242508

75 Spaldin NA. Search for ferromagnetism in transition-metal-doped piezoelectric ZnO. Phys Rev B, 2004, 69: 125201

76 Gopal P, Spaldin NA. Magnetic interactions in transition-metaldoped ZnO: an ab initio study. Phys Rev B, 2006, 74: 094418

77 Yuhas BD, Fakra S, Marcus MA, Yang PD. Probing the local coordination environment for transition metal dopants in zinc oxide nanowires. Nano Lett, 2007, 7: 905-909

78 Yao T, Yan WS, Sun ZH, et al. Magnetic property and spatial occupation of Co dopants in $\mathrm{Zn}_{0.98} \mathrm{Co}_{0.02} \mathrm{O}$ nanowires. J Phys Chem C, 2009, 113: 14114-14118

$79 \mathrm{Yu}$ JH, Liu XY, Kweon KE, et al. Giant Zeeman splitting in nucleation-controlled doped CdSe: $\mathrm{Mn}^{2+}$ quantum nanoribbons. Nat Mater, 2010, 9: 47-53

80 Segura-Ruiz J, Martinez-Criado G, Chu MH, Geburt S, Ronning C. Nano-X-ray absorption spectroscopy of single Co-implanted $\mathrm{ZnO}$ nanowires. Nano Lett, 2011, 11: 5322-5326

81 Yan WS, Liu QH, Wang C, et al. Realizing ferromagnetic coupling in diluted magnetic semiconductor quantum dots. J Am Chem Soc, 2014, 136: 1150-1155

82 Sun ZH, Yang XY, Wang C, et al. Graphene activating room-temperature ferromagnetic exchange in cobalt-doped $\mathrm{ZnO}$ dilute magnetic semiconductor quantum dots. ACS Nano, 2014, 8: 10589 10596

83 Sun YF, Sun ZH, Gao S, et al. Fabrication of flexible and freestanding zinc chalcogenide single layers. Nat Commun, 2012, 3: 1057

84 Sun YF, Cheng H, Gao S, et al. Freestanding tin disulfide single-layers realizing efficient visible-light water splitting. Angew Chem Int Ed, 2012, 51: 8727-8731

85 Sun YF, Cheng H, Gao S, et al. Atomically thick bismuth selenide freestanding single layers achieving enhanced thermoelectric energy harvesting. J Am Chem Soc, 2012, 134: 20294-20297

86 Sun YF, Liu QH, Gao S, et al. Pits confined in ultrathin cerium(IV) oxide for studying catalytic centers in carbon monoxide oxidation. Nat Commun, 2013, 4: 2899

87 Cheng WR, He JF, Yao T, et al. Half-unit-cell alpha- $\mathrm{Fe}_{2} \mathrm{O}_{3}$ semicon- 
ductor nanosheets with intrinsic and robust ferromagnetism. J Am Chem Soc, 2014, 136: 10393-10398

88 Sun YF, Sun ZH, Gao S, et al. All-surface-atomic-metal chalcogenide sheets for high-efficiency visible-light photoelectrochemical water splitting. Adv Energ Mater, 2014, 4: doi: 10.1002/aenm.201300611

89 Yao T, Sun ZH, Li YY, et al. Insights into initial kinetic nucleation of gold nanocrystals. J Am Chem Soc, 2010, 132: 7696-7701

90 Mesu JG, van der Eerden AM, de Groot FM, Weckhuysen BM. Synchrotron radiation effects on catalytic systems as probed with a combined in-situ UV-Vis/XAFS spectroscopic setup. J Phys Chem B, 2005, 109: 4042-4047

91 Mesu JG, Beale AM, de Groot FM, Weckhuysen BM. Probing the influence of X-rays on aqueous copper solutions using time-resolved in situ combined video/X-ray absorption near-edge/ultraviolet-visible spectroscopy. J Phys Chem B, 2006, 110: 17671-17677

92 Yao T, Liu SJ, Sun ZH, et al. Probing nucleation pathways for morphological manipulation of platinum nanocrystals. J Am Chem Soc, 2012, 134: 9410-9416

93 Ohyama J, Teramura K, Higuchi Y, et al. An in situ quick XAFS spectroscopy study on the formation mechanism of small gold nanoparticles supported by porphyrin-cored tetradentate passivants. Phys Chem Chem Phys, 2011, 13: 11128-11135

94 Ohyama J, Teramura K, Higuchi Y, et al. In situ observation of nucleation and growth process of gold nanoparticles by quick XAFS spectroscopy. ChemPhysChem, 2011, 12: 127-131

95 Tanaka T, Ohyama J, Teramura K, Hitomi Y. Formation mechanism of metal nanoparticles studied by XAFS spectroscopy and effective synthesis of small metal nanoparticles. Catal Today, 2012, 183: 108-118

96 Harada M, Einaga H. In situ XAFS studies of Au particle formation by photoreduction in polymer solutions. Langmuir, 2007, 23 : 6536-6543

97 Harada M, Inada Y. In situ time-resolved XAFS studies of metal particle formation by photoreduction in polymer solutions. Langmuir, 2009, 25: 6049-6061

98 Harada M, Tamura N, Takenaka M. Nucleation and growth of metal nanoparticles during photoreduction using in situ time-resolved SAXS analysis. J Phys Chem C, 2011, 115: 14081-14092

99 Harada M, Kamigaito Y. Nucleation and aggregative growth process of platinum nanoparticles studied by in situ quick XAFS spectroscopy. Langmuir, 2012, 28: 2415-2428

100 Banares MA. Operando spectroscopy: the knowledge bridge to assessing structure-performance relationships in catalyst nanoparticles. Adv Mater, 2011, 23: 5293-5301

101 Tada M, Murata S, Asakoka T, et al. In situ time-resolved dynamic surface events on the $\mathrm{Pt} / \mathrm{C}$ cathode in a fuel cell under operando conditions. Angew Chem Int Ed, 2007, 46: 4310-4315

102 Uemura Y, Inada Y, Bando KK, et al. Core-shell phase separation and structural transformation of $\mathrm{Pt}_{3} \mathrm{Sn}$ alloy nanoparticles support- ed on gamma- $\mathrm{Al}_{2} \mathrm{O}_{3}$ in the reduction and oxidation processes characterized by in situ time-resolved XAFS. J Phys Chem C, 2011, 115: 5823-5833

103 Tada M, Uemura Y, Bal R, et al. In situ time-resolved DXAFS for the determination of kinetics of structural changes of H-ZSM-5-supported active Re-cluster catalyst in the direct phenol synthesis from benzene and $\mathrm{O}_{2}$. Phys Chem Chem Phys, 2010, 12: 5701-5706

104 Ishiguro N, Saida T, Uruga T, et al. Operando time-resolved X-ray absorption fine structure study for surface events on a $\mathrm{Pt}_{3} \mathrm{Co} / \mathrm{C}$ cathode catalyst in a polymer electrolyte fuel cell during voltageoperating processes. ACS Catal, 2012, 2: 1319-1330

105 Wang XQ, Hanson JC, Frenkel AI, Kim JY, Rodriguez JA. Timeresolved studies for the mechanism of reduction of copper oxides with carbon monoxide: complex behavior of lattice oxygen and the formation of suboxides. J Phys Chem B, 2004, 108: 13667-13673

106 Wang Q, Hanson JC, Frenkel AI. Solving the structure of reaction intermediates by time-resolved synchrotron X-ray absorption spectroscopy. J Chem Phys, 2008, 129: 234502

107 Wang X, Rodriguez JA, Hanson JC, Perez M, Evans J. In situ time-resolved characterization of $\mathrm{Au}-\mathrm{CeO}_{2}$ and $\mathrm{AuO}_{x}-\mathrm{CeO}_{2}$ catalysts during the water-gas shift reaction: presence of $\mathrm{Au}$ and $\mathrm{O}$ vacancies in the active phase. J Chem Phys, 2005, 123: 221101

108 Frenkel AI, Rodriguez JA, Chen JGG. Synchrotron techniques for in situ catalytic studies: capabilities, challenges, and opportunities. ACS Catal, 2012, 2: 2269-2280

109 Rodriguez JA, Hanson JC, Stacchiola D, Senanayake SD. In situ/operando studies for the production of hydrogen through the watergas shift on metal oxide catalysts. Phys Chem Chem Phys, 2013, 15: 12004-12025

Acknowledgements This research was supported by the National Natural Science Foundation of China (11135008, 11175184 and 11475176), and the Foundation for Innovative Research Groups of the National Natural Science Foundation of China (11321503). We would like to express our gratitude to Profs. Kunquan Lu, Xinyi Zhang, Yi Xie, Ziyu Wu, and Tiandou $\mathrm{Hu}$ for valuable discussions and encouragements. We also express our thanks to Dr. Zhiyun Pan, Dr. Yong Jiang, Dr. Yongfu Sun, Dr. Jingfu He, Mr. Zhi Xie, Mr. Bo He and Miss Fengchun Hu for fruitful collaborations. We appreciate the National Synchrotron Radiation Laboratory (NSRL), Beijing Synchrotron Radiation Facility (BSRF), and Shanghai Synchrotron Radiation Facility (SSRF) for the valuable beamtimes that we used in our experiments.

Author contributions Wei S initiated and guided the whole project. Sun Z, Liu Q, Yao T, and Yan W surveyed the literatures, discussed the content, and wrote the manuscript

Conflict of interest The authors declare that they have no conflict of interest. 

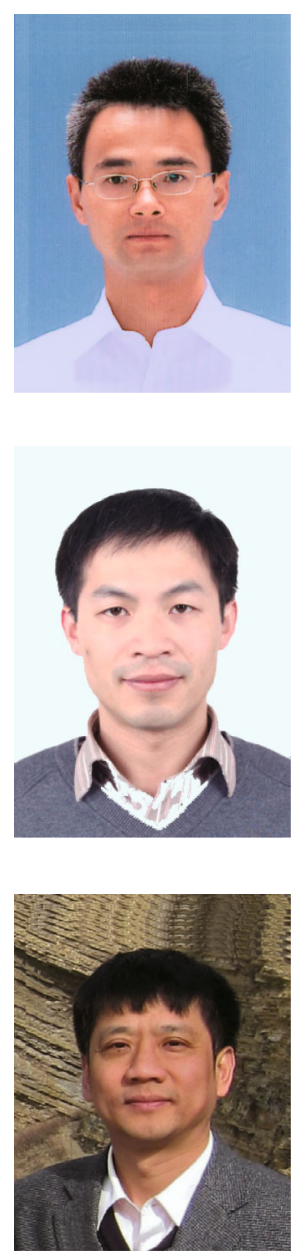

Zhihu Sun received his PhD degree from University of Science and Technology of China (USTC) in 2005. After post-doctoral research at National Institute of Advanced Industrial Science and Technology of Japan, he became an associate professor of National Synchrotron Radiation Laboratory at USTC. His research interest includes the applications of synchrotron radiation techniques to low-dimensional nanostructures and magnetic semiconductor materials.

Qinghua Liu received his PhD degree in 2009 from USTC. He is an associate professor at National Synchrotron Radiation Laboratory at USTC. His research interest includes on X-ray absorption spectroscopy and photocatalysis.

Shiqiang Wei is a full professor at USTC. He received his PhD degree from USTC in 1992. After working as a senior researcher at National Institute of Advanced Industrial Science and Technology of Japan, he was a gainer of the "One-hundred Talents Project" of Chinese Academy of Sciences in 1998, won the "Chinese National Science Fund for Distinguished Young Scholars" in 2007, and became the chief scientist at National Synchrotron Radiation Laboratory of China in 2008. His current research interest covers the development of synchrotron radiation techniques and their applications in advanced function materials.

中文摘要 X射线吸收精细结构谱学 $(\mathrm{XAFS})$ 技术是近 40 年来同步辐射领域最为重要的实验技术之一,在物理、化学、生物、材料和能 源科学等诸多领域得到了广泛应用. 本文简要综述了XAFS技术在纳米材料科学研究中的应用. 首先介绍了XAFS的基本原理、实验方 法及数据分析处理过程, 进一步通过对XAFS技术在应用于纳米材料研究中的一些代表性工作进行概述, 如金属与半导体纳米颗粒、 催化剂、核壳结构、二维超薄纳米片, 以及原位XAFS技术在研究纳米材料的原位成核/生长过程和纳米催化剂在工作状态下的中间态 表征等, 展示了XAFS技术在纳米材料领域对其原子和电子结构表征的强大能力. 